\title{
TRACING CYCLIC HOMOLOGY PAIRINGS UNDER TWISTING OF GRADED ALGEBRAS
}

\author{
SAYAN CHAKRABORTY \\ Mathematisches Institut, Westfälische Wilhelms-Universität Münster \\ Einsteinstr. 62, 48149 Münster, Germany \\ MAKOTO YAMASHITA \\ Department of Mathematics, Ochanomizu University \\ Otsuka 2-1-1, Bunkyo, Tokyo, Japan \\ Current address: Department of Mathematics, University of Oslo
}

\begin{abstract}
We give a description of cyclic cohomology and its pairing with $K$-groups for 2-cocycle deformation of algebras graded over discrete groups. The proof relies on a realization of monodromy for the Gauss-Manin connection on periodic cyclic cohomology in terms of the cup product action of group cohomology.
\end{abstract}

\section{INTRODUCTION}

Cyclic homology theory as initiated by Tsygan 48 and Connes 8 provides a natural extension of de Rham theory for manifolds to objects of noncommutative geometry, which are represented by noncommutative algebras. The main subject of this paper is the cyclic cohomology for deformation of algebras graded by some discrete group $G$, with deformation parameter given by exponential of a 2cocycle on $G$. This deformation scheme has many good analogies with the theory of (formal) deformation quantization of Poisson manifolds, but at the same time gives a concrete example of strict deformation in the sense of Rieffel, meaning that we can specialize the deformation parameter to concrete numbers and represent the deformed algebra on Hilbert spaces. The most fundamental example in this setting is the celebrated noncommutative torus $\mathbb{T}_{\theta}^{2}$, which is represented by a twisted group algebra of $\mathbb{Z}^{2}$, and is also a deformation quantization of $\mathbb{T}^{2}$ with respect to the translation invariant Poisson structure.

Our goal is to understand: (1) the cyclic cohomology of such algebras as abstract linear spaces, and (2) the pairing with $K$-groups / cyclic homology groups for deformed algebras, in terms of those for the original algebra and the cohomology of $G$. For the case of the group algebra of $G=\mathbb{Z}^{2}$ (which leads to the noncommutative torus), these were carried out in $8,13,37,41]$, but beyond the commutative (or finite, in which case this deformation is trivial) case, the full understanding is limited to only a handful of cases. A notable case is the crossed product of $\mathbb{Z}^{2}$ by a finite subgroup of $\mathrm{SL}_{2}(\mathbb{Z})$, where relatively comprehensive description of $K$-groups, cyclic cohomology groups, and their pairings for twisted group algebras are known 5, 39, 40,51,52. Let us also mention that another interesting work from a geometric viewpoint is carried out by Mathai 31] for the standard traces of twisted group algebras for many interesting classes of groups.

Regarding the point (1) above, usual computation of cyclic cohomology starts with the computation of Hochschild cohomology groups, which serve as analogue of the spaces of differential forms. Once this is done, the cyclic cohomology groups can be obtained using the map induced by Rinehart-Connes operator, playing the role of the exterior derivative. However, while the end result of computation, particularly the periodic cyclic cohomology groups tend to be invariant under deformation of algebra structures, the intermediate Hochschild cohomology is very sensitive to deformation. This is already apparent in the

E-mail addresses: sayan.c@uni-muenster.de, yamashita.makoto@ocha.ac.jp (makotoy@math.uio.no).

Date: December 2, 2018.

Key words and phrases. graded algebra, cyclic homology, cocycle twisting, K-theory. 
case of noncommutative torus, and there is little hint for the more general case of deformation of graded algebras by 2-cocycles, even just for the twisted group algebras.

Our approach, instead, is based on the Gauss-Manin connection for periodic cyclic (co)homology introduced by Getzler [14]. The monodromy for this connection, if it makes sense, will give a natural isomorphism between the periodic cyclic cohomology of the deformed algebra and that of the original one. This plays a particularly powerful role in the setting of Poisson deformation quantization, and nicely explains the relation between the Poisson homology of a Poisson manifold and the cyclic homology of the deformed algebra. Motivated by this, our viewpoint could be phrased as follows: the group algebra of $G$ represents a quantum group, which is (up to "Poincaré dual") homotopically modeled on the classifying spaces for centralizers of torsion elements of $G$. A 2-cocycle $\omega_{0}$ on $G$ represents an (invariant) "Poisson bivector" on this quantum group. A G-grading on an algebra corresponds to an action of this quantum group, by which we can transport the "Poisson bivector" and related structures.

A new challenge in the setting of strict deformation is that the Gauss-Manin connection is intrinsically defined on the infinite dimensional space of periodic cyclic (co)chains, and there is no general theorem to integrate it. Developing the second named author's previous work [54] on "invariant" cyclic cocycles, we solve this issue by identifying the action of connection operator up to chain homotopy by the cup product action of group cohomology.

As it turns out, this formalism also fits well with recent developments in the operator algebraic $K$ theory of such algebras [12,53], where one considers the $C([0,1])$-algebra whose fiber at $t \in[0,1]$ is the deformation with respect to the rescaled 2-cocycle $e^{t \omega_{0}}$ where $\omega_{0}$ is an $\sqrt{-1} \mathbb{R}$-valued 2-cocycle. If $G$ satisfies the Baum-Connes conjecture with coefficients, the evaluation at each $t$ induces isomorphism of $K$-groups, which basically solves the problem (2). On the purely algebraic side, the image of " $C^{\infty}$ sections" in periodic cyclic homology are flat with respect to the Gauss-Manin connection. This allows us to express the pairing of $K$-groups and cyclic cohomology of the deformed algebra in terms of the original data and the action of group cohomology. As an application, we look at the twisted group algebras of crystallographic groups. The $K$-theory of untwisted algebra was studied in detail by Lück and his collaborators $11,26,29$, and our result give a description of cyclic cohomology and its pairing with $K$-groups for twisted group $C^{*}$-algebras for such groups.

The paper is organized as follows. In Section 1, we recall the basic terminology and prepare notations for algebraic notions. In Section 2, exploiting the comparison of Hochschild cohomology of group algebra and group cohomology [6], we construct traces on twisted group algebras which are supported on the conjugacy classes of torsion elements. This leads to isomorphism between the cyclic cohomology groups of general 2-cocycle deformation of graded algebras over such conjugacy classes, solving the problem (1). Section 3 is the main part of the paper, where we compute the monodromy of the Gauss-Manin connection with respect to the identification established in Section 2. We collect further analytic preliminaries in Section 4. In Section 5, we establish $K$-theoretic comparison between Fréchet algebraic models and operator algebraic models, as a basis to compute the pairing of operator algebraic $K$-theory and cyclic cohomology of "smooth" algebras. In Section 6, we apply our general theory to twisted algebras of crystallographic groups.

Acknowledgements. The authors would like to thank Wolfgang Lück and Ryszard Nest for fruitful comments. They also thank the following programs / grants for their support which enabled collaboration for this paper: Simons - Foundation grant 346300 and the Polish Government MNiSW 2015-2019 matching fund; The Isaac Newton Institute for Mathematical Sciences for the programme Operator algebras: subfactors and their applications. This work was supported by: EPSRC grant number EP/K032208/1 and DFG (SFB 878). M.Y. thanks the operator algebra group at University of Münster for their hospitality during his stay, and Yasu Kawahigashi for financial support (JSPS KAKENHI Grant Number 15H02056).

\section{Algebraic PREliminaries}

1.1. Noncommutative calculus. Let us first review the fundamental concepts in cyclic homology theory. Basic references are 10, 27, 49.

Let $A$ be a unital $\mathbb{C}$-algebra, and put $\mathrm{C}_{n}(A)=A^{\otimes n+1}$. Recall that the Hochschild differential $\mathrm{C}_{n}(A) \rightarrow$ $\mathrm{C}_{n-1}(A)$ is given by

$$
b\left(a_{0} \otimes \cdots \otimes a_{n}\right)=\sum_{i=0}^{n-1}(-1)^{i} a_{0} \otimes \cdots \otimes a_{i} a_{i+1} \otimes \cdots \otimes a_{n}+(-1)^{n} a_{n} a_{0} \otimes a_{1} \otimes \cdots \otimes a_{n-1},
$$


while the Rinehart-Connes differential $\mathrm{C}_{n}(A) \rightarrow \mathrm{C}_{n+1}(A)$ is

$$
\begin{aligned}
B\left(a_{0} \otimes \cdots \otimes a_{n}\right)=\sum_{i=0}^{n}(-1)^{n i}\left(1 \otimes a_{n+1-i} \otimes \cdots \otimes a_{n} \otimes a_{0} \otimes \cdots \otimes a_{n-i}\right. \\
\left.\quad+(-1)^{n} a_{n-i} \otimes 1 \otimes a_{n+1-i} \otimes \cdots \otimes a_{n} \otimes a_{0} \otimes \cdots \otimes a_{n-i-1}\right) .
\end{aligned}
$$

The associated cyclic complex is given by

$$
\mathrm{CC}_{n}(A)=\mathrm{C}_{n}(A) \oplus \mathrm{C}_{n-2}(A) \oplus \cdots \oplus \mathrm{C}_{n-2\left\lfloor\frac{n}{2}\right\rfloor}(A),
$$

endowed with the differential $b-B: \mathrm{CC}_{n}(A) \rightarrow \mathrm{CC}_{n-1}(A)$. Its homology $\mathrm{HC}_{n}(A)=\mathrm{H}_{n}\left(\mathrm{CC}_{*}(A)\right)$ is the $n$-th cyclic homology of $A$. The periodic cyclic homology $\operatorname{HP}_{*}(A)$ is defined as the homology of $\mathbb{Z} / 2 \mathbb{Z}$ graded complex $\left(\mathrm{CP}_{*}(A), b-B\right)$ with $\mathrm{CP}_{0}(A)=\prod_{k=0}^{\infty} \mathrm{C}_{2 k}(A)$ and $\mathrm{CP}_{1}(A)=\prod_{k=0}^{\infty} \mathrm{C}_{2 k+1}(A)$. The linear dual spaces $\mathrm{C}^{n}(A)=\left(\mathrm{C}_{n}(A)\right)^{\prime}$ form the dual complex, with transpose maps $b^{\#}$ and $B^{\#}$. Then the cyclic cohomology $\mathrm{HC}^{*}(A)$ is simply defined as the cohomology of $\left(\mathrm{CC}^{n}(A), b^{\#}-B^{\#}\right)$, while the periodic cyclic cohomology $\operatorname{HP}^{*}(A)$ is defined as the cohomology of $\mathbb{Z} / 2 \mathbb{Z}$-graded complex $\left(\mathrm{CP}^{*}(A), b^{\#}-B^{\#}\right)$ with $\mathrm{CP}^{0}(A)=\bigoplus_{k=0}^{\infty} \mathrm{C}^{2 k}(A)$ and $\mathrm{CP}^{1}(A)=\bigoplus_{k=0}^{\infty} \mathrm{C}^{2 k+1}(A)$.

The $S$-map $S: \mathrm{CC}_{n}(A) \rightarrow \mathrm{CC}_{n-2}(A)$ is the projection onto the second direct summand of the decomposition $\mathrm{CC}_{n}(A)=\mathrm{C}_{n}(A) \oplus \mathrm{CC}_{n-2}(A)$. This induces a map $\mathrm{HC}_{n}(A) \rightarrow \mathrm{HC}_{n-2}(A)$ on the homology, again denoted by $S$.

The reduced Hochschild complex of $A$ is given by $\overline{\mathrm{C}}_{n}(A)=A \otimes \bar{A}^{\otimes n}$, where $\bar{A}=A / \mathbb{C}$. The kernel of quotient map $\mathrm{C}_{n}(A) \rightarrow \overline{\mathrm{C}}_{n}(A)$ is stable under $b$, and is contractible (in the sense of complex). Thus, $\left(\overline{\mathrm{C}}_{*}, b\right)$ also computes the Hochschild homology of $A$, and its linear dual complex $\overline{\mathrm{C}}^{*}(A)$ computes the Hochschild cohomology. On this model $B$ is given by

$$
B: \overline{\mathrm{C}}_{n}(A) \rightarrow \overline{\mathrm{C}}_{n+1}(A), \quad a_{0} \otimes \cdots \otimes a_{n} \rightarrow \sum_{i=0}^{n}(-1)^{n i} 1 \otimes a_{i} \otimes \cdots \otimes a_{n} \otimes a_{0} \otimes \cdots \otimes a_{i-1} .
$$

The A-valued Hochschild n-cochains are by definition the elements of $\mathrm{C}^{n}(A ; A)=\operatorname{Hom}_{\mathbb{C}}\left(A^{\otimes n}, A\right)$. The direct sum with degree shift $\mathrm{C}^{*-1}(A ; A)$ is a graded pre-Lie algebra by partial composition $D \circ D^{\prime}$. The product map of $A$ gives an element $\mu \in \mathrm{C}^{2}(A ; A)$, and the graded commutator

$$
\delta(D)=[\mu, D]=\mu \circ D-(-1)^{m-1} D \circ \mu
$$

for $D \in \mathrm{C}^{m}(A ; A)$ can be computed as

$$
\begin{array}{r}
a_{1} \otimes \cdots \otimes a_{m+1} \mapsto(-1)^{m-1}\left(a_{1} D\left(a_{2} \otimes \cdots \otimes a_{m+1}\right)+\sum_{k=1}^{m}(-1)^{k} D\left(a_{1} \otimes \cdots \otimes a_{k} a_{k+1} \otimes \cdots \otimes a_{m+1}\right)\right. \\
\left.+(-1)^{m+1} D\left(a_{1} \otimes \cdots \otimes a_{m}\right) a_{m+1}\right),
\end{array}
$$

which is the 'degree shift' of the Hochschild differential. Given such a cochain $D \in \mathrm{C}^{m}(A ; A)$, following 14 (but in the convention of [54]) we consider the operators

$$
\begin{aligned}
& \mathbf{B}_{D}\left(a_{0} \otimes \cdots \otimes a_{n}\right) \\
& \quad=\sum_{0 \leq j \leq k \leq n-m}(-1)^{n(j+1)+(m-1)(k-j)} 1 \otimes a_{j+1} \otimes \cdots \otimes D\left(a_{k+1} \otimes \cdots \otimes a_{k+m}\right) \otimes \cdots \otimes a_{n} \otimes a_{0} \otimes \cdots \otimes a_{j} .
\end{aligned}
$$

and

$$
\mathbf{b}_{D}\left(a_{0} \otimes \cdots \otimes a_{n}\right)=(-1)^{m n} D\left(a_{n-m+1} \otimes \cdots \otimes a_{n}\right) a_{0} \otimes \cdots \otimes a_{n-m} .
$$

acting on $\mathrm{C}_{*}(A)$. Then $\iota_{D}=\mathbf{b}_{D}-\mathbf{B}_{D}$ is called the interior product by $D$. Another important operation is the Lie derivative

$$
\begin{aligned}
L_{D}\left(a_{0} \otimes \ldots \otimes a_{n}\right) & =\sum_{j=0}^{n-m}(-1)^{(m-1)(j+1)} a_{0} \otimes \cdots \otimes D\left(a_{j+1} \otimes \cdots \otimes a_{j+m}\right) \otimes \cdots \otimes a_{n} \\
& +\sum_{j=n-m+1}^{n}(-1)^{n(n-j)} D\left(a_{j+1} \otimes \cdots \otimes a_{0} \otimes a_{1} \otimes \cdots \otimes a_{j+m-n-1}\right) \otimes a_{j+m-n} \otimes \cdots \otimes a_{j} .
\end{aligned}
$$

Let us denote by $\overline{\mathrm{C}}^{n}(A ; A)$ the space of cochains with $D\left(a_{1} \otimes \cdots \otimes a_{n}\right)=0$ whenever $a_{i}=1$ for some $i$. This is a subcomplex for $\delta$, and $\iota_{D}$ and $L_{D}$ are operators on $\overline{\mathrm{C}}_{*}(A)$ when $D$ is in this space. We then have the following Cartan homotopy formula relating these two kinds of operators. 
Proposition 1.1 (14:42, 54, Proposition 4]). When $D \in \overline{\mathrm{C}}^{m}(A ; A)$, we have

$$
\left[b-B, \iota_{D}\right]=L_{D}-\iota_{\delta(D)}
$$

where the left hand side is the graded commutator $(b-B) \iota_{D}-(-1)^{m} \iota_{D}(b-B)$ in $\operatorname{End}\left(\overline{\mathrm{C}}_{*}(A)\right)$.

Let us recall the formalism of Gauss-Manin connection on periodic cyclic (co)homology [14]. Suppose $\left(\mu_{t}\right)_{t \in I}$ is a 'smooth' family of associative product structures parametrized by $I=[0,1]$ with common multiplicative unit on $A$. We then consider $\Gamma^{\infty}(I ; A)$ which is, as a linear space, the union of smooth functions from $I$ into finite dimensional subspaces of $A$. The product structure is given pointwise by $\mu_{t}$, that is $\left(f * f^{\prime}\right)(t)=\mu_{t}\left(f(t), f^{\prime}(t)\right)$ for $f, f^{\prime} \in \Gamma^{\infty}(I ; A)$ (so we require a regularity condition on $\mu_{t}$ to make this well-defined).

Then $\partial_{t}$ is a Hochschild 1-cochain of $\Gamma^{\infty}(I ; A)$ which is not a derivation unless $\mu_{t}$ is constant in $t$. Note that $\delta\left(\partial_{t}\right)=\left[\mu, \partial_{t}\right]$ is, up to sign, equal to the time derivative $\dot{\mu}_{t}$ of the product structure. Hence the Cartan homotopy formula says

$$
\left[b-B, \iota_{\partial_{t}}\right]=L_{\partial_{t}}+\iota_{\dot{\mu}_{t}} .
$$

as operators on $\overline{\mathrm{C}}_{*}\left(\Gamma^{\infty}(I ; A)\right)$. Moreover, $L_{\partial_{t}}$ is the natural extension of $\partial_{t}$ to chains by the Leibniz rule. Rewriting this again as $\partial_{t}$, we obtain an operator $\nabla=\partial_{t}+\iota_{\dot{\mu}_{t}}$ acting on the space $\Gamma^{\infty}\left(I ; \mathrm{CP}_{*}(A)\right)$, which is the Gauss-Manin connection on periodic cyclic homology. Being the commutator with $b-B$, it commutes with $b-B$, and hence descends to an operator on $\Gamma^{\infty}\left(I ; \operatorname{HP}_{*}(A)\right)$. Moreover, if $x \in \operatorname{HP}_{*}\left(\Gamma^{\infty}(I ; A)\right)$, its image $\mathrm{ev}(x)$ in $\Gamma^{\infty}\left(I ; \mathrm{HP}_{*}(A)\right)$ satisies $\nabla \mathrm{ev}(x)=0$ by 1.2 . Thus, morally speaking, if we choose an element $x_{0} \in \mathrm{HP}_{*}(A)$ with respect to the product structure $\mu_{0}$, a solution $\left(x_{t}\right)_{t}$ to the differential equation $\nabla x_{t}=0$ describes the lifting of $x_{0}$ to $\mathrm{HP}_{*}\left(\Gamma^{\infty}(I ; A)\right)$, and the same can be said for the elements of $\operatorname{HP}^{*}(A)$. However, this is a difficult task in general since we are considering a differential equation on an infinite dimensional space, and we will solve this equation in a slightly different way.

1.2. Cup product in cyclic cohomology. Let $A$ and $A^{\prime}$ be two unital $\mathbb{C}$-algebras. On the one hand, the complex $\mathrm{C}_{*}\left(A \otimes A^{\prime}\right)$ can be regarded as a product $\mathrm{C}_{*}(A) \times \mathrm{C}_{*}\left(A^{\prime}\right)$ of mixed modules [27, Chapter 4], that is

together with differentials

$$
\left(\mathrm{C}_{*}(A) \times \mathrm{C}_{*}\left(A^{\prime}\right)\right)_{p}=\mathrm{C}_{p}(A) \otimes \mathrm{C}_{p}\left(A^{\prime}\right),
$$

$$
\begin{gathered}
b_{\mathrm{C}_{*}(A) \times \mathrm{C}_{*}\left(A^{\prime}\right)}=b \otimes b: \mathrm{C}_{p}(A) \otimes \mathrm{C}_{p}\left(A^{\prime}\right) \rightarrow \mathrm{C}_{p-1}(A) \otimes \mathrm{C}_{p-1}\left(A^{\prime}\right), \\
B_{\mathrm{C}_{*}(A) \times \mathrm{C}_{*}\left(A^{\prime}\right)}=B \otimes B: \mathrm{C}_{p}(A) \otimes \mathrm{C}_{p}\left(A^{\prime}\right) \rightarrow \mathrm{C}_{p+1}(A) \otimes \mathrm{C}_{p+1}\left(A^{\prime}\right) .
\end{gathered}
$$

On the other, usual tensor product of complexes $\mathrm{C}_{*}(A) \otimes \mathrm{C}_{*}\left(A^{\prime}\right)$ is again a mixed module by differentials $b \otimes$ id $+(-1)^{*}$ id $\otimes b$ and $B \otimes$ id $+(-1)^{*}$ id $\otimes B$. Taking the dual of total $(b, B)$-bicomplex of these, we obtain cyclic cohomology groups $\mathrm{HC}^{*}\left(\mathrm{C}_{*}(A) \times \mathrm{C}_{*}\left(A^{\prime}\right)\right)=\mathrm{HC}^{*}\left(A \otimes A^{\prime}\right)$ and $\mathrm{HC}^{*}\left(\mathrm{C}_{*}(A) \otimes \mathrm{C}_{*}\left(A^{\prime}\right)\right)$. If $\phi$ (resp. $\psi$ ) is a cyclic $m$-cocycle on $A$ (resp. cyclic $n$-cocycle on $A^{\prime}$ ), their tensor product $\phi \otimes \psi$ gives a $(m+n)$-cyclic cocycle on $\mathrm{C}_{*}(A) \otimes \mathrm{C}_{*}\left(A^{\prime}\right)$, or an class in $\mathrm{HC}^{m+n}\left(\mathrm{C}_{*}(A) \otimes \mathrm{C}_{*}\left(A^{\prime}\right)\right)$.

There is a map Sh of total complexes of $\mathrm{C}_{*}(A) \otimes \mathrm{C}_{*}(A)$ into that of $\mathrm{C}_{*}(A) \times \mathrm{C}_{*}\left(A^{\prime}\right)$ called the shuffle product, which is a quasi-isomorphism for $b-B$. Concretely, it is given as follows.

When $(p, q)$ is a pair of nonnegative integers, we let $S_{p, q}$ denote the set

$$
\left\{\sigma \in S_{p+q} \mid \sigma(1)<\cdots<\sigma(p), \sigma(p+1)<\cdots<\sigma(p+q)\right\},
$$

called the set of $(p, q)$-shuffles. Let $p+q=n$, and let $\operatorname{sh}_{p, q}$ denote the linear map of $C_{p}(A) \otimes C_{q}\left(A^{\prime}\right)$ into $C_{n}(A) \otimes C_{n}\left(A^{\prime}\right)$ defined by

$$
\operatorname{sh}_{p, q}(a, b)=\sum_{\sigma \in S_{p, q}} s_{\sigma(p+1)} \cdots s_{\sigma(p+q)}(a) \otimes s_{\sigma(1)} \cdots s_{\sigma(p)}(b) .
$$

Here, $s_{i}$ is the $i$-th degeneracy operator ('insert 1 at the $i$-th place'). The direct sum of these maps for $p+q=n$ defines a map $\left(C_{*}(A) \otimes C_{*}\left(A^{\prime}\right)\right)_{n} \rightarrow\left(C_{*}(A) \times C_{*}\left(A^{\prime}\right)\right)_{n}$, denoted by $\operatorname{sh}_{n}$.

Next, a $(p, q)$-cyclic shuffle is any element $\sigma$ of $S_{p+q}$ which can be written as $\sigma=\mu \circ t_{1 \ldots p}^{a} t_{(p+1) \cdots(p+q)}^{b}$ for $\mu \in S_{p, q}$ and $a, b \in \mathbb{N}$, which also satisfies $\sigma(1)<\sigma(p+1)$. Here, $t_{1 \ldots p}$ is the cyclic permutation $t_{1 \cdots p}(k)=k(\bmod p)$ on $\{1, \ldots, p\}$, and $t_{(p+1) \cdots(p+q)}$ is the one $t_{(p+1) \cdots(p+q)}(k)=k+1(\bmod q)$ on $\{p+1, \ldots, p+q\}$. We put $S_{p, q}^{\text {cyc }}$ the set of $(p, q)$-cyclic shuffles. One then obtains a map $\perp_{p, q}$ from $C_{p}(A) \otimes C_{q}\left(A^{\prime}\right)$ to $C_{p+q}(A) \otimes C_{p+q}\left(A^{\prime}\right)$, given by

$$
x \perp_{p, q} y=\sum_{\sigma=\mu t_{1 \ldots p}^{a} t_{(p+1) \cdots(p+q)}^{b} \in S_{p, q}^{\text {cyc }}}(-1)^{|s|} s_{\mu(p+1)} \cdots s_{\mu(p+q)} t_{p}^{a}(x) \otimes s_{\mu(1)} \cdots s_{\mu(p)} t_{q}^{b}(y),
$$


and a linear map $\operatorname{sh}_{p, q}^{\prime}$ of $C_{p}(A) \otimes C_{q}\left(A^{\prime}\right)$ into $C_{n+2}(A) \otimes C_{n+2}\left(A^{\prime}\right)$ by

$$
\operatorname{sh}_{p, q}^{\prime}(a, b)=s(a) \perp_{p+1, q+1} s(b),
$$

where $s$ is the extra degeneracy operator ('insert 1 at the 0 -th place'). Then, the direct sum of the $\operatorname{sh}_{p, q}^{\prime}$ for $p+q=n$ defines a map $\left(C_{*}(A) \otimes C_{*}\left(A^{\prime}\right)\right)_{n} \rightarrow\left(C_{*}(A) \times C_{*}\left(A^{\prime}\right)\right)_{n+2}$ denoted by $\operatorname{sh}_{n}^{\prime}$.

Finally, $\mathrm{Sh}$ is defined as $\mathrm{Sh}=\mathrm{sh}-\mathrm{sh}^{\prime}$. This is a morphism of the total complexes of the mixed complexes (it commutes with $b-B$ ), which is actually a quasi-isomorphism 27, Thteorem 4.3.8]. Its transpose map is again a quasi-isomorphism and, we obtain a cyclic cohomology class $[\phi \cup \psi]$ in $\operatorname{HC}^{m+n}\left(A \otimes A^{\prime}\right)$ corresponding to $\phi \otimes \psi$, called the cup product. Of course, this class depends only on those of $\phi$ and $\psi$.

Remark 1.2. There is another formulation $\phi \# \psi$, which goes through the correspondence between the cyclic cocycles and the closed graded traces on differential graded algebras $\left(\Omega^{*}, d: \Omega^{*} \rightarrow \Omega^{*+1}\right)$ with homomorphism $A \rightarrow \Omega^{0}$ [8. Then $\phi \# \psi$ is given by the graded tensor product of the objects involved. When $\phi\left(\right.$ resp. $\psi$ ) is a cyclic $m$-cocycle on $A$ (resp. cyclic $n$-cocycle on $A^{\prime}$ ), we have

$$
\frac{1}{(m+n) !}[\phi \# \psi]=\frac{1}{m ! n !}[\phi \cup \psi]
$$

in $\mathrm{HC}^{m+n}\left(A \otimes A^{\prime}\right)$.

1.3. Standard complex for group cohomology. Let $G$ be a discrete group, and $M$ be a commutative group (either $\mathbb{R}, \mathbb{C}, \mathbb{T}=\mathbb{R} / \mathbb{Z}$, or $\mathbb{C}^{\times}$for our purposes). The group cohomology $\mathrm{H}^{*}(G ; M)$ is equal to the cohomology of the standard complex $\left(\mathrm{C}^{*}(G ; M), d\right)$, where $\mathrm{C}^{n}(G ; M)=\operatorname{Map}\left(G^{n}, M\right)$ and $d: \mathrm{C}^{n}(G ; M) \rightarrow$ $\mathrm{C}^{n+1}(G ; M)$ is given by

$$
\begin{aligned}
& d \phi\left(g_{1}, \ldots, g_{n+1}\right)=\phi\left(g_{2}, \ldots, g_{n+1}\right) \\
& +\sum_{i=1}^{n}(-1)^{i} \phi\left(g_{1}, \ldots, g_{i} g_{i+1}, \ldots, g_{n+1}\right)+(-1)^{n+1} \phi\left(g_{1}, \ldots, g_{n}\right) .
\end{aligned}
$$

We formally put $\mathrm{C}^{0}(G ; M)=\operatorname{Map}(\{*\}, M)=M$ and $d$ to be the zero map from $\mathrm{C}^{0}(G ; M)$ to $\mathrm{C}^{1}(G ; M)$. Consequently, $\mathrm{H}^{0}(G ; M)=M$ and the 2-cocycles are the 2-point functions satisfying

$$
\phi(h, k)+\phi(g, h k)=\phi(g, h)+\phi(g h, k) .
$$

Obviously, $\mathrm{C}^{*}(G)=\mathrm{C}^{*}(G ; \mathbb{C})$ is the dual complex of $\mathrm{C}_{*}(G)=\left(\mathbb{C}\left[G^{n}\right]\right)_{n=0}^{\infty}$, endowed with differential $d: \mathrm{C}_{n+1}(G) \rightarrow \mathrm{C}_{n}(G)$ analogous to that of $\mathrm{C}^{*}(G)$.

If $G$ is a finite group, one has $\mathrm{H}^{n}(G ; \mathbb{C})=0$ for $n>0$. This is a consequence of the identification $\mathrm{H}^{*}(G ; \mathbb{C}) \simeq \operatorname{Ext}_{G}^{*}(\mathbb{C}, \mathbb{C})$ and the semisimplicity of $G$-vector spaces, but more concretely we can use Shapiro's lemma type argument: for example, given any 2-cocycle $\omega_{0}$, one defines $\psi \in \mathrm{C}^{1}(G ; \mathbb{C})$ by

$$
\psi(g)=\frac{1}{|G|} \sum_{h \in G} \omega_{0}(h, g) .
$$

Then one can verify $d \psi(g, h)=\omega_{0}(g, h)$ by concrete computation using the 2-cocycle identity.

A group cochain $\phi \in \mathrm{C}^{n}(G ; M)$ is normalized if

$$
\phi\left(g_{1}, \ldots, g_{i}, e, g_{i+1}, \ldots, g_{n-1}\right)=0 \quad(0 \leq i \leq n-1)
$$

and $\phi\left(g_{1}, \ldots, g_{n}\right)=0$ whenever $g_{1} \cdots g_{n}=e$. (Note that this requirement is stronger than the usual one found for example in $[4$.

Proposition 1.3 (22, 28, Section 4]). When $K$ is a field of characteristic zero and $n>0$, any $K$-valued $n$-cocycle is cohomologous to a normalized one.

While the above does not immediately apply to $\mathbb{T}$ - or $\mathbb{C}^{\times}$-valued cocycles, a direct argument still shows that the 2-cocycles with these coefficients can still be normalized. Let us also note the following cyclicity of normalized cocycles.

Proposition 1.4. Let $\phi$ be a normalized n-cocycle on $G$. Then we have

$$
\phi\left(\left(g_{1} \cdots g_{n}\right)^{-1}, g_{1}, \ldots, g_{n-1}\right)=(-1)^{n} \phi\left(g_{1}, \ldots, g_{n}\right) .
$$

Proof. Compute the value of $0=d \phi$ on $\left(\left(g_{1} \cdots g_{n}\right)^{-1}, g_{1}, \ldots, g_{n}\right)$ and use the normalization condition. 
1.4. Cyclic cocycles on group algebra. Let us now describe the cyclic homology theory for group algebra $\mathbb{C}[G]$, which is essentially from $[6]$. Its adaptation to cyclic cohomologies is already explained in 18, but we will later need explicit constructions in this section.

For each $x \in G$, let us denote the centralizer of $x$ in $G$ by $C_{G}(x)$, and the conjugacy class of $x$ by $\operatorname{Ad}_{G}(x)$. Let $\mathrm{C}_{n}^{x}(\mathbb{C}[G])$ be the subspace of $\mathrm{C}_{n}(\mathbb{C}[G])=\mathbb{C}[G]^{\otimes n+1}$ spanned by those $g_{0} \otimes \cdots \otimes g_{n}$ such that $g_{0} \cdots g_{n} \in \operatorname{Ad}_{G}(x)$. The spaces $\left(\mathrm{C}_{n}^{x}(\mathbb{C}[G])\right)_{n=0}^{\infty}$ give a subcomplex for $b$ and $B$, which leads to the direct sum decomposition

$$
\mathrm{HH}_{*}(\mathbb{C}[G])=\bigoplus_{x \in G \backslash_{\mathrm{Ad}} G} \mathrm{H}_{*}\left(\mathrm{C}_{*}^{x}(\mathbb{C}[G]), b\right), \quad \mathrm{HC}_{*}(\mathbb{C}[G])=\bigoplus_{x \in G \backslash_{\mathrm{Ad}} G} \mathrm{H}_{*}\left(\mathrm{CC}\left(\mathrm{C}_{*}^{x}(\mathbb{C}[G])\right), b-B\right),
$$

where $\mathrm{CC}\left(\mathrm{C}_{*}^{x}(\mathbb{C}[G])\right)$ is the direct summand of $\mathrm{CC}(\mathbb{C}[G])$ formed by the spaces $\mathrm{C}_{n}^{x}(\mathbb{C}[G])$, which can be regarded as the cyclic complex associated with the cyclic module $\mathrm{C}_{*}^{x}(\mathbb{C}[G])$, see [27]. Dualizing this, we also obtain the direct product decomposition

$$
\mathrm{HH}^{*}(\mathbb{C}[G])=\prod_{x \in G \backslash_{\mathrm{Ad}} G} \mathrm{H}^{*}\left(\mathrm{C}_{*}^{x}(\mathbb{C}[G])^{\prime}, b^{\#}\right), \quad \mathrm{HC}^{*}(\mathbb{C}[G])=\prod_{x \in G \backslash_{\mathrm{Ad}} G} \mathrm{H}^{*}\left(\mathrm{CC}\left(\mathrm{C}_{*}^{x}(\mathbb{C}[G])\right)^{\prime}, b^{\#}-B^{\#}\right) .
$$

Let us denote the factors labeled by $x$ by $\mathrm{HH}^{*}(\mathbb{C}[G])_{x}$ and $\mathrm{HC}^{*}(\mathbb{C}[G])_{x}$. We want to describe them in terms of group cohomology.

From now on let us fix $x$, and also choose and fix $g^{y}=g_{x}^{y} \in G$ such that $\left(g^{y}\right)^{-1} x g^{y}=y$ for each element $y$ in $\operatorname{Ad}_{G}(x)$.

Let $\left(g_{0}, \ldots, g_{n}\right) \in G^{n+1}$ be such that $g_{0} \cdots g_{n} \in \operatorname{Ad}_{G}(x)$. We put

$$
y_{i}=g_{i} \cdots g_{n} g_{0} \cdots g_{i-1} \text {. }
$$

Note that these elements are also in $\operatorname{Ad}_{G}(x)$.

Lemma 1.5. The elements $g^{y_{i}} g_{i}\left(g^{y_{i+1}}\right)^{-1}$ for $0 \leq i<n$, and $g^{y_{n}} g_{n}\left(g^{y_{0}}\right)^{-1}$ are in $C_{G}(x)$.

Proof. This follows from direct calculation using $x g^{y}=g^{y} y$ and $y_{i} g_{i}=g_{i} y_{i+1}$.

We next consider the map

$$
\Xi: \mathrm{C}_{n}^{x}(\mathbb{C}[G]) \rightarrow \mathrm{C}_{n}\left(C_{G}(x)\right), \quad g_{0} \otimes \cdots \otimes g_{n} \mapsto\left(g^{y_{1}} g_{1}\left(g^{y_{2}}\right)^{-1}, \ldots, g^{y_{n}} g_{n}\left(g^{y_{0}}\right)^{-1}\right) .
$$

For $n=0$, we put $\Xi\left(g_{0}\right)=*$, where we formally write $\left(C_{G}\right)^{0}=\{*\}$.

Proposition 1.6. The map $\Xi$ intertwines the Hochschild differential $b$ on $\left(\mathrm{C}_{n}^{x}(\mathbb{C}[G])\right)_{n=0}^{\infty}$ and $d$ on $\mathrm{C}_{*}\left(C_{G}(x)\right)$.

Proof. We can compare (1.1) and 1.3) directly term by term.

Consequently, any cocycle on $C_{G}(x)$ induces a Hochschild cocycle on $\mathbb{C}[G]$ which is supported on the conjugacy class of $x$. For example, the trivial class represented by $1 \in \mathbb{C}=\mathrm{C}^{0}\left(C_{G}(x)\right)$ corresponds to the trace $\tau^{x}(f)=\delta_{\operatorname{Ad}_{G}(x)}(f)=\sum_{g \in \operatorname{Ad}_{G}(x)} f(g)$ on $\mathbb{C}[G]$.

In fact, the above proposition corresponds to the fact that $\Xi$ is induced by a map of cyclic sets from $\Gamma_{*}(G, x)$ to $B_{*}\left(C_{G}(x), x\right)$ in the notation of 27]. A cyclic set is a simplicial set

$$
\left(\left(X_{n}\right)_{n=0}^{\infty} ;\left(d_{i}^{n}: X_{n} \rightarrow X_{n-1}\right)_{i=0}^{n},\left(s_{i}^{n}: X_{n} \rightarrow X_{n+1}\right)_{i=0}^{n}\right)
$$

endowed with $t_{n}: X_{n} \rightarrow X_{n}$ satisfying certain compatibility conditions [27, Chapter 6]. On the one hand, $\Gamma_{n}(G, x)$ is the natural bases of $\mathrm{C}_{n}^{x}(\mathbb{C}[G])$. On the other, the simplicial set $B_{n}(G)=G^{n}$ is a cyclic set by

$$
t_{n}\left(g_{1}, \ldots, g_{n}\right)=\left(\left(g_{1} \cdots g_{n}\right)^{-1}, g_{1}, \cdots, g_{n-1}\right) .
$$

The cyclic set $B_{*}\left(C_{G}(x), x\right)$ has the same underlying simplicial structure as $B_{*}\left(C_{G}(x)\right)$, but the cyclic structure $t_{n}$ is given by $\left(x\left(g_{1} \cdots g_{n}\right)^{-1}, g_{1}, \cdots, g_{n-1}\right)$ instead.

Proposition 1.7. The map $\Xi$ induces an isomorphism $\mathrm{HH}^{*}(\mathbb{C}[G])_{x} \simeq \mathrm{H}^{*}\left(C_{G}(x) ; \mathbb{C}\right)$.

Proof. This corresponds to the first half of [6, Theorem I']. More concretely, consider the chain map $\Upsilon$ from $\mathrm{C}_{*}\left(C_{G}(x)\right)$ to $\mathrm{C}_{*}^{x}(\mathbb{C}[G])$ defined by

$$
\Upsilon\left(g_{1}, \ldots, g_{n}\right)=\left(g_{1} \cdots g_{n}\right)^{-1} x \otimes g_{1} \otimes \cdots \otimes g_{n} .
$$

Then, on the one hand, $\Xi \Upsilon$ is equal to the identity map on $\mathrm{C}_{*}\left(C_{G}(x)\right)$. On the other, $\Upsilon \Xi$ can be computed as

$$
g_{0} \otimes \cdots \otimes g_{n} \mapsto g^{y_{0}} g_{0}\left(g^{y_{1}}\right)^{-1} \otimes g^{y_{1}} g_{1}\left(g^{y_{2}}\right)^{-1} \otimes \ldots \otimes g^{y_{n}} g_{n}\left(g^{y_{0}}\right)^{-1} .
$$


This map is homotopic to the identity on $\mathrm{C}_{*}^{x}(\mathbb{C}[G])$ as follows (cf. 15, Section III.2]). Put

$$
\begin{aligned}
& \theta_{0}\left(g_{0} \otimes \cdots \otimes g_{n}\right)=g^{y_{0}} g_{0} \otimes g_{1} \otimes \cdots \otimes g_{n} \otimes\left(g^{y_{0}}\right)^{-1}, \\
& \theta_{1}\left(g_{0} \otimes \cdots \otimes g_{n}\right)=g^{y_{0}} g_{0} \otimes g_{1} \otimes \cdots \otimes g_{n-1} \otimes\left(g^{y_{n}}\right)^{-1} \otimes g^{y_{n}} g_{n}\left(g^{y_{0}}\right)^{-1}, \ldots \\
& \theta_{n}\left(g_{0} \otimes \cdots \otimes g_{n}\right)=g^{y_{0}} g_{0} \otimes\left(g^{y_{1}}\right)^{-1} \otimes g^{y_{1}} g_{1}\left(g^{y_{2}}\right)^{-1} \otimes \cdots \otimes g^{y_{n}} g_{n}\left(g^{y_{0}}\right)^{-1} .
\end{aligned}
$$

Then the alternating sum

$$
h_{n}=\sum_{i=0}^{n}(-1)^{n+i+1} \theta_{i}: \mathrm{C}_{n}^{x}(\mathbb{C}[G]) \rightarrow \mathrm{C}_{n+1}^{x}(\mathbb{C}[G])
$$

satisfies $b h_{n}+h_{n-1} b=\mathrm{id}-\Upsilon \Xi$. Consequently, $\Xi$ and $\Upsilon$ induce isomorphism of cohomology between the dual complexes.

Lemma 1.8. Let $\phi$ be a normalized $n$-cocycle on $C_{G}(x)$. The Hochschild cocycle $\phi \Xi$ on $\mathbb{C}[G]$ descends to $\overline{\mathrm{C}}_{n}(\mathbb{C}[G])$.

Proof. We need to check

$$
\phi \Xi\left(h_{0} \otimes \cdots \otimes h_{i} \otimes e \otimes h_{i+1} \otimes \cdots \otimes h_{n-1}\right)=0 \quad(0 \leq i \leq n-1)
$$

whenever $h_{0} \cdots h_{n-1} \in \operatorname{Ad}_{G}(x)$.

Putting $\left(g_{0}, \ldots, g_{n}\right)=\left(h_{0}, \ldots, h_{i}, e, h_{i+1}, \ldots, h_{n-1}\right)$, one has $y_{i+1}=y_{i+2}$ in the above notation and hence the $(i+1)$-th component of $\Xi\left(g_{0}, \ldots, g_{n}\right)$ is $e$. By the normalization condition on $\phi$, we have the required vanishing.

Let $N_{G}(x)$ be the quotient of $C_{G}(x)$ by the subgroup $\langle x\rangle$ generated by $x$.

Proposition 1.9. Let $\phi$ be a normalized n-cocycle on $N_{G}(x)$. The induced cocycle $\tilde{\phi}$ on $C_{G}(x)$ satisfies $\tilde{\phi} \Xi B=0$.

Proof. We want to show the vanishing of $\phi\left(g^{y_{j}} g_{j}\left(g^{y_{j+1}}\right)^{-1}, \ldots, g^{y_{j-1}} g_{j-1}\left(g^{y_{j}}\right)^{-1}\right)$ for each $j$. Using the cyclicity of Proposition 1.4 this is equal to

$$
(-1)^{n} \phi\left(\left(g^{y_{j}} y_{j}\left(g^{y_{j}}\right)^{-1}\right)^{-1}, g^{y_{j}} g_{j}\left(g^{y_{j+1}}\right)^{-1}, \ldots, g^{y_{j-2}} g_{j-2}\left(g^{y_{j-1}}\right)^{-1}\right) .
$$

Since $\left(g^{y_{j}} y_{j}\left(g^{y_{j}}\right)^{-1}\right)^{-1}$ is equal to $x^{-1}$, which becomes $e$ in $N_{G}(x)$, the normalization condition implies the vanishing of this term.

It follows that normalized cocycles on $N_{G}(x)$ define reduced cyclic cocycles on $\mathbb{C}[G]$, that is, functionals on $\overline{\mathrm{C}}_{n}(\mathbb{C}[G])$ annihilated by both $b^{\#}$ and $B^{\#}$. We next want to understand if they survive in the periodic cyclic cohomology.

First let us suppose that $x$ is of infinite order. In this case one has $\operatorname{HC}^{n}(\mathbb{C}[G])_{x} \simeq \mathrm{H}^{n}\left(N_{G}(x) ; \mathbb{C}\right)$. Moreover, the Gysin maps associated with the fibration of classifying spaces $S^{1}=B\langle x\rangle \rightarrow B C_{G}(x) \rightarrow$ $B N_{G}(x)$ defines a linear map $f: \mathrm{H}^{n}\left(N_{G}(x) ; \mathbb{C}\right) \rightarrow \mathrm{H}^{n+2}\left(N_{G}(x) ; \mathbb{C}\right)$ for each $k$, which is identified with the $S$-map $\operatorname{HC}^{n}(\mathbb{C}[G])_{x} \rightarrow \mathrm{HC}^{n+2}(\mathbb{C}[G])_{x}[6$, Section IV; 27, Chapter 7]. It follows that the periodic cyclic cohomology class associated with a normalized $n$-cocycle $\phi$ on $N_{G}(x)$ only depends on its image in the inductive limit $\lim _{k} \mathrm{H}^{n+2 k}\left(N_{G}(x) ; \mathbb{C}\right)$.

Remark 1.10. The above Gysin map can be described as follows. The Hochschild-Serre spectral sequence associated with the extension $\langle x\rangle \rightarrow C_{G}(x) \rightarrow N_{G}(x)$ has the $E_{2}$-page $\mathrm{H}^{p}\left(N_{G}(x) ; \mathrm{H}^{q}(\langle x\rangle ; \mathbb{C})\right)$, which are concentrated on $q=0,1$. The $E_{2}$-differential

$$
d_{2}: \mathrm{H}^{p}\left(N_{G}(x) ; \mathrm{H}^{1}(\langle x\rangle ; \mathbb{C})\right) \rightarrow \mathrm{H}^{p+2}\left(N_{G}(x) ; \mathrm{H}^{0}(\langle x\rangle ; \mathbb{C})\right)
$$

can be identified with maps $\mathrm{H}^{p}\left(N_{G}(x) ; \mathbb{C}\right) \rightarrow \mathrm{H}^{p+2}\left(N_{G}(x) ; \mathbb{C}\right)$ which is exactly the Gysin map. The $d_{2}$ is also directly described by the cup product with the extension class 11. Namely, the central extension $\langle x\rangle \rightarrow C_{G}(x) \rightarrow N_{G}(x)$ corresponds to a class in $\mathrm{H}^{2}\left(N_{G}(x) ;\langle x\rangle\right)$. Identifying $\langle x\rangle$ with $\mathbb{Z}$ and embedding it in $\mathbb{C}$, we obtain a class $c_{G ; x}$ in $\mathrm{H}^{2}\left(N_{G}(x) ; \mathbb{C}\right)$. The cup product by $c_{G ; x}$ is $d_{2}$. It follows that $\lim _{\longrightarrow k} \mathrm{H}^{n+2 k}\left(N_{G}(x) ; \mathbb{C}\right)$ is trivial if and only if $c_{G ; x}$ represents a nilpotent element in the group cohomology $\overrightarrow{\text { of }} N_{G}(x)$.

In particular, if $N_{G}(x)$ has bounded cohomology degree, the conjugacy class of $x$ does not contribute in $\operatorname{HP}^{*}(\mathbb{C}[G])$. Let us denote the class of discrete groups such that any subgroup has bounded cohomology degree by $\mathcal{C}$. 
Lemma 1.11. The class $\mathcal{C}$ contains finite groups, finitely generated commutative groups, free groups, fundamental groups of acyclic manifolds, and is closed under extensions.

Proof. One obtains the statement about extensions from Hochschild-Serre spectral sequence.

When $x$ is of finite order, the situation is much simpler. The finiteness of $\langle x\rangle$ implies $\mathrm{H}^{q}(\langle x\rangle ; \mathbb{C})=0$ for $q>0$, hence the Hochschild-Serre spectral sequence implies $\mathrm{H}^{n}\left(C_{G}(x) ; \mathbb{C}\right) \simeq \mathrm{H}^{n}\left(N_{G}(x)\right.$; $\left.\mathbb{C}\right)$. Usually the following proposition is stated in the homological form, but the proof carries over without change since it is about the simplicial structure of the underlying cyclic set $B_{*}\left(C_{G}(x) ; x\right)$.

Proposition 1.12 ([6. Section IV; 27. Chapter 7]). Suppose that $x \in G$ is of finite order. Then the $S$-map $\mathrm{HC}^{n}(\mathbb{C}[G])_{x} \rightarrow \mathrm{HC}^{n+2}(\mathbb{C}[G])_{x}$ is identified with the canonical inclusion as the direct summand

$$
\bigoplus_{k=0}^{\left\lfloor\frac{n}{2}\right\rfloor} \mathrm{H}^{n-2 k}\left(N_{G}(x) ; \mathbb{C}\right) \rightarrow \bigoplus_{k=0}^{\left\lfloor\frac{n}{2}\right\rfloor+1} \mathrm{H}^{n-2 k+2}\left(N_{G}(x) ; \mathbb{C}\right),
$$

hence one has

$$
\mathrm{HP}^{*}(\mathbb{C}[G])_{x} \simeq \bigoplus_{k \equiv * \bmod 2} \mathrm{H}^{k}\left(N_{G}(x) ; \mathbb{C}\right)=\bigoplus_{k \equiv * \bmod 2} \mathrm{H}^{k}\left(C_{G}(x) ; \mathbb{C}\right)
$$

1.5. Action of group cohomology on graded algebras. Let us next review ring and module structure on cyclic cohomology of group algebras and graded algebras, mainly from [18,54.

Let $A$ be a $G$-graded algebra, that is, $A$ decomposes as a direct sum $\bigoplus_{g \in G} \overline{A_{g}}$ satisfying $1 \in A_{e}$ and $A_{g} A_{h} \subset A_{g h}$. This can be encoded as a coaction homomorphism

$$
\alpha: A \rightarrow A \otimes \mathbb{C}[G], \quad a \mapsto a \otimes g \quad\left(a \in A_{g}\right) .
$$

There is also a direct product decomposition

$$
\operatorname{HP}^{*}(A)=\prod_{x \in G \backslash_{\mathrm{Ad}} G} \operatorname{HP}^{*}(A)_{x}
$$

analogous to the case of group algebra.

We then have an action of $\operatorname{HP}^{*}(\mathbb{C}[G])$ on $\operatorname{HP}^{*}(A)$, given by $\phi \triangleleft \psi=\alpha^{*}(\phi \cup \psi)$ with $\phi \in \operatorname{HP}^{*}(A)$ and $\psi \in \operatorname{HP}^{*}(\mathbb{C}[G])$. In particular, with $A=\mathbb{C}[G]$, we obtain a ring structure on $\operatorname{HP}^{*}(\mathbb{C}[G])$. The trace $\tau^{x}=\delta_{\operatorname{Ad}_{G}(x)}$ is an idempotent, and for general $G$-graded $A$, it acts on $\operatorname{HP}^{*}(A)$ as the projection onto $\operatorname{HP}^{*}(A)_{x}$.

Proposition 1.13. Let $x$ be of finite order. The factor $\mathrm{HP}^{*}(\mathbb{C}[G])_{x}$ is a subring, whose product structure is isomorphic to the cup product on $\mathrm{H}^{*}\left(C_{G}(x) ; \mathbb{C}\right)$.

Proof. That $\operatorname{HP}^{*}(\mathbb{C}[G])_{x}$ is a subring follows from the fact that $\delta_{\operatorname{Ad}_{G}(x)}$ is a central idempotent for the above product. It remains to identify the product structure.

On the one hand, by [54, Corollary 9], the cup product on $\operatorname{HC}^{*}\left(B_{*}\left(N_{G}(x)\right)\right)$ coincides with that of $\mathrm{H}^{*}\left(N_{G}(x)\right)$ under the standard comparison map. On the other, there is a map of cyclic modules from $\mathrm{C}_{*}^{x}(\mathbb{C}[G])$ to $\mathbb{C}\left[B_{*}\left(N_{G}(x)\right)\right]$ by composing $\Xi$ with the natural surjection $\mathbb{C}\left[B_{*}\left(C_{G}(x), x\right)\right] \rightarrow$ $\mathbb{C}\left[B_{*}\left(N_{G}(x)\right)\right]$. The cup product is natural for map of cyclic modules, hence we obtain the assertion.

Remark 1.14. The above proposition is, at least to some extent, known to experts. For example 18 contains a very similar module structure based on $\phi \# \psi$. But we feel that some details, such as a precise form of product structure on $\mathrm{HC}^{k}(\mathbb{C}[G])_{x} \simeq \mathrm{H}^{k}\left(N_{G}(x) ; \mathbb{C}\right) \oplus \mathrm{H}^{k-2}\left(N_{G}(x) ; \mathbb{C}\right) \oplus \cdots$, and the compatibility under the periodicity map $S$, need some additional elaboration.

Proposition 1.15. Let $x$ be an element of infinite order in $G$. The $S$-map $\operatorname{HC}^{n}(A)_{x} \rightarrow \mathrm{HC}^{n+2}(A)_{x}$ is equal to the cup product by the extension class $c_{G ; x} \in \mathrm{HC}^{2}(\mathbb{C}[G])_{x}=\mathrm{H}^{2}\left(N_{G}(x) ; \mathbb{C}\right)$.

Proof. This is proved in [18, Theorem 6.2] under a different convention of the $S$-map and cup product (see Remark 1.2). Let us give an alternate shorter proof: the $S$-map in our convention is the cup product by a class in $\mathrm{HC}^{2}(\mathbb{C})$ represented by the functional $\nu$ on $\mathrm{C}_{2}(\mathbb{C})$ given by $v(1 \otimes 1 \otimes 1)=-\frac{1}{2}$. Identifying $\mathrm{HC}^{n}(A)_{x} \otimes \mathrm{HC}^{2}(\mathbb{C})$ with $\mathrm{HC}^{n}(A)_{x} \otimes \mathrm{HC}^{2}(\mathbb{C}) \otimes \mathrm{HC}^{0}(\mathbb{C}[G])_{x}$ and using the associativity of cup product, we can identify $S(\phi)$ with $\phi \cup S\left(1_{\mathrm{H}^{*}\left(N_{G}(x)\right)}\right)$ for $\phi \in \mathrm{HC}^{n}(A)_{x}$. By Remark 1.10, we know that $c_{G ; x}$ represents $S\left(1_{\mathrm{H}^{*}\left(N_{G}(x)\right)}\right)$.

Corollary 1.16 (cf. 18 , Corollary 6.6]). If $\operatorname{HP}^{*}(\mathbb{C}[G])_{x}$ is trivial, then $\operatorname{HP}^{*}(A)_{x}$ is also trivial. 
1.6. Deformation of graded algebras. Let $A$ be a $G$-graded algebra, and $\omega$ be a normalized $\mathbb{C}^{\times}$valued 2-cocycle. We can then define a new associative product on $A$ by

$$
a *_{\omega} b=\omega\left(g_{1}, g_{2}\right) a b
$$

for homogeneous elements $a \in A_{g_{1}}$ and $b \in A_{g_{2}}$. Thanks to the normalization condition on $\omega, 1_{A}$ is still the unit for this product. We denote this algebra by $A_{\omega}$, and its element corresponding to $a \in A$ by $a^{(\omega)}$.

There is an embedding of algebra $A_{\omega} \rightarrow A \otimes \mathbb{C}_{\omega}[G]$ given by

$$
a^{(\omega)} \mapsto a \otimes \lambda_{g}^{(\omega)} \quad\left(a \in A_{g}\right) .
$$

\section{INDUCING TRACES ON TWISTED ALGEBRAS}

Let $\omega_{0}(g, h)$ be a $\mathbb{C}$-valued normalized 2-cocycle on $G$. We consider the twisted group algebras for the family of $\mathbb{C}^{\times}$-valued 2-cocycles $\omega^{t}(g, h)=e^{t \omega_{0}(g, h)}$, so that the new product is $g *_{t} h=\omega^{t}(g, h) g h$.

We want to 'deform' the trace $\tau=\delta_{\operatorname{Ad}_{G}(x)}$ on $\mathbb{C}[G]$ to a one on $\mathbb{C}_{\omega^{t}}[G]$. Note that when $g h \in \operatorname{Ad}_{G}(x)$, we have

$$
\delta_{\operatorname{Ad}_{G}(x)}\left(g *_{t} h\right)=\omega^{t}(g, h), \quad \delta_{\operatorname{Ad}_{G}(x)}\left(h *_{t} g\right)=\omega^{t}(h, g),
$$

but these factors do not need to be equal. We want to correct this situation by setting

$$
\tau_{\omega^{t}}^{x}(g)=e^{t \xi(g)} \delta_{\operatorname{Ad}_{G}(x)}(g)
$$

for some function $\xi$ on $\operatorname{Ad}_{G}(x)$. Then $\tau_{\omega^{t}}^{x}$ becomes a trace if we have

$$
\omega_{0}(g, h)+\xi(g h)=\omega_{0}(h, g)+\xi(h g)
$$

This means that the bilinear extension of $\omega_{0}(g, h)-\omega_{0}(h, g)$ is the Hochschild coboundary of the linear extension of $-\xi$. Let us put $\omega_{0}^{a}(g, h)=\omega_{0}(g, h)-\omega_{0}(h, g)$.

Lemma 2.1. The bilinear extension of $\omega_{0}^{a}$ represents a 1 -cocycle in the complex $\left(\mathrm{C}_{*}^{x}(\mathbb{C}[G])^{\prime}, b\right)$.

Proof. We need to show

$$
\omega_{0}^{a}(g h, k)-\omega_{0}^{a}(g, h k)+\omega_{0}^{a}(k g, h)=0
$$

whenever $g h k \in \operatorname{Ad}_{G}(x)$. Expanding the definition of $\omega_{0}^{a}$, this is the same as

$$
\omega_{0}(g h, k)+\omega_{0}(h k, g)+\omega_{0}(k g, h)=\omega_{0}(k, g h)+\omega_{0}(g, h k)+\omega_{0}(h, k g) .
$$

Adding $\omega_{0}(g, h)+\omega_{0}(h, k)+\omega_{0}(k, g)$ to both sides and using the cocycle identity, we indeed obtain the equality.

Lemma 2.2. Let $H$ and $K$ be subgroups of $G$ such that any $h \in H$ and $k \in K$ satisfy $h k=k h$. Then $\omega_{0}^{a}$ is an additive bicharacter on $H \times K$, i.e. $\omega_{0}^{a}(h, k)$ is a homomorphism into $\mathbb{C}$ for both of the variables $h$ and $k$.

Proof. Since $\omega_{0}^{a}(k, h)=-\omega_{0}^{a}(h, k)$, it is enough to prove the additivity in $k$.

We first claim that $\omega_{0}^{a}(h, k)=-\omega_{0}^{a}\left(k h, k^{-1}\right)$. This amounts to showing

$$
\omega_{0}(h, k)+\omega_{0}\left(k h, k^{-1}\right)=\omega_{0}(k, h)+\omega_{0}\left(k^{-1}, k h\right) .
$$

Using the commutativity of $h$ and $k$, and the 2-cocycle property, the left hand side becomes $\omega_{0}\left(k, k^{-1}\right)+$ $\omega_{0}(h, e)$, while the right hand side becomes $\omega_{0}\left(k^{-1}, k\right)+\omega_{0}(e, h)$. Since $\omega_{0}$ is normalized, these are equal to 0 .

Now, let us verify the claim, which by the above is equivalent to

$$
\omega_{0}^{a}\left(k_{1} k_{2} h, k_{2}^{-1} k_{1}^{-1}\right)=\omega_{0}^{a}\left(k_{2} h, k_{2}^{-1}\right)+\omega_{0}^{a}\left(k_{1} h, k_{1}^{-1}\right) .
$$

Putting $s_{1}=k_{1} k_{2} h, s_{2}=k_{2}^{-1}$, and $s_{3}=k_{1}^{-1}$, this can be written as

$$
\omega_{0}\left(s_{1}, s_{2} s_{3}\right)+\omega_{0}\left(s_{2}, s_{3} s_{1}\right)+\omega_{0}\left(s_{3}, s_{1} s_{2}\right)=\omega_{0}\left(s_{2} s_{3}, s_{1}\right)+\omega_{0}\left(s_{3} s_{1}, s_{2}\right)+\omega_{0}\left(s_{1} s_{2}, s_{3}\right) .
$$

By the same argument as in the proof of Lemma 2.1, this is indeed the case.

Proposition 2.3. Suppose $x$ is of finite order. Then there is a function $\xi: \operatorname{Ad}_{G}(x) \rightarrow \mathbb{C}$ such that $\omega_{0}^{a}=-b^{\#} \xi$. 
Proof. We first note that $\left(\Upsilon^{\#} \omega_{0}^{a}\right)(g)=\omega_{0}^{a}\left(g^{-1} x, g\right)$ is zero. Indeed, using the 2-cocycle identity and normalization condition, we have

$$
\omega_{0}^{a}\left(g^{-1} x, g\right)=\omega_{0}\left(g^{-1} x, g\right)-\omega_{0}\left(g, g^{-1} x\right)=-\omega_{0}\left(x, g^{-1}\right)+\omega_{0}\left(g^{-1}, x\right)=-\omega_{0}^{a}\left(x, g^{-1}\right) .
$$

Lemma 2.2 for $\langle x\rangle$ and $C_{G}(x)$ implies that this is 0 , since any homomorphism from $\langle x\rangle$ to $\mathbb{C}$ must be trivial.

Then, by the homotopy formula $b h_{1}+h_{0} b=\mathrm{id}-\Upsilon \Xi$ from Section 1.3 and $b^{\#} \omega_{0}^{a}=0$ from Lemma 2.1. we see that $\xi=-h_{0}^{\#} \omega_{0}^{a}$ is indeed a solution.

Example 2.4. Suppose we have $G=\mathbb{Z}^{2} \rtimes \mathbb{Z}_{3}$, where the generator 1 of $\mathbb{Z}_{3}$ acts on $\mathbb{Z}^{2}$ by the matrix

$$
A=\left[\begin{array}{cc}
-1 & -1 \\
1 & 0
\end{array}\right]
$$

In the following we denote elements of $G$ by triples $(a, b, i)$ for $a, b \in \mathbb{Z}$ and $i \in \mathbb{Z}_{3}$. This group has the following conjugacy classes.

$$
\left\{(a, b, 0) \mid(a, b) \in \mathbb{Z}_{3} . z\right\} \text { for } z \in \mathbb{Z}_{3} \backslash \mathbb{Z}^{2},
$$

$$
\begin{array}{lll}
\{(a, b, 1) \mid a \equiv b \bmod 3\}, & \{(a, b, 1) \mid a \equiv b+1 \bmod 3\}, & \{(a, b, 1) \mid a \equiv b+2 \bmod 3\}, \\
\{(a, b, 2) \mid a \equiv b \bmod 3\}, & \{(a, b, 2) \mid a \equiv b+1 \bmod 3\}, & \{(a, b, 2) \mid a \equiv b+2 \bmod 3\} .
\end{array}
$$

We take the 2-cocycle $\omega_{0}$ on $\mathbb{Z}^{2}$ given by $\omega_{0}((a, b),(c, d))=a d-b c$, and extend it to $G$ by

$$
\omega_{0}((a, b, g),(c, d, h))=\omega_{0}((a, b), g \cdot(c, d)) \quad\left(a, b, c, d \in \mathbb{Z}, g, h \in \mathbb{Z}_{3}\right) .
$$

This represents a generator of $\mathrm{H}^{2}(G ; \mathbb{C})$. We then put $\omega_{\theta}(g, h)=\exp \left(-\sqrt{-1} \frac{\theta}{2} \omega_{0}(g, h)\right)$. Let us present a concrete computation which follows from the above general consideration for the case of $x=(0,0,1)$, which is of order three. We have

$$
\begin{aligned}
& \omega_{\theta}((a, b, 0),(c, d, 1))=\omega_{\theta}((a, b),(c, d))=\exp \left(-\frac{\sqrt{-1} \theta}{2}(a d-b c)\right), \\
& \omega_{\theta}((c, d, 1),(a, b, 0))=\omega_{\theta}((c, d),(-a-b, a))=\exp \left(-\frac{\sqrt{-1} \theta}{2}(c a+a d+b d)\right) .
\end{aligned}
$$

Thus, in the twisted group algebra $\mathbb{C}_{\omega_{\theta}}[G]$, we have

$$
\begin{aligned}
& \lambda_{(a, b, 0)}^{\left(\omega_{\theta}\right)} \lambda_{(c, d, 1)}^{\left(\omega_{\theta}\right)}=\exp \left(-\frac{\sqrt{-1} \theta}{2}(a d-b c)\right) \lambda_{(a+c, b+d, 1)}^{\left(\omega_{\theta}\right)}, \\
& \lambda_{(c, d, 1)}^{\left(\omega_{\theta}\right)} \lambda_{(a, b, 0)}^{\left(\omega_{\theta}\right)}=\exp \left(-\frac{\sqrt{-1} \theta}{2}(c a+a d+b d)\right) \lambda_{(c-a-b, d+a, 1)}^{\left(\omega_{\theta}\right)} .
\end{aligned}
$$

In particular, $\delta_{\operatorname{Ad}_{G}(x)}$ is not a trace, but a correction like

$$
\tau_{\omega_{\theta}}^{x}\left(\lambda_{(a, b, 1)}\right)=\exp \left(-\frac{\sqrt{-1} \theta\left(a^{2}+a b+b^{2}\right)}{6}\right) \delta_{\operatorname{Ad}_{G}(x)}(a, b, 1)
$$

is (see Section 6.1 for comparison with [5]). Let us relate the extra factor appearing here to the above general discussion. On the one hand, using the chain homotopy $h_{i}$ in $(1.4), \omega_{0}^{a} b=0$ and $\omega_{0}^{a} \Upsilon=0$ implies that $\xi$ can be taken as $-\omega_{0}^{a} h_{0}$. On the other, for $y=(a, b, 1) \in \operatorname{Ad}_{G}(x)$, the element $g^{y}=\left(\frac{b-a}{3}, \frac{-a-2 b}{3}, 0\right)$ satisfies $\left(g^{y}\right)^{-1} x g^{y}=y$. Thus $\xi$ is given by

$$
\xi\left(g_{0}\right)=-\omega_{0}^{a} h_{0}\left(g_{0}\right)=\omega_{0}^{a} \theta_{0}\left(g_{0}\right)=\omega_{0}\left(g^{g_{0}} g_{0},\left(g^{g_{0}}\right)^{-1}\right)-\omega_{0}\left(\left(g^{g_{0}}\right)^{-1}, g^{g_{0}} g_{0}\right)=\frac{a^{2}+a b+b^{2}}{3}
$$

for $g_{0}=(a, b, 1)$ with $a \equiv b \bmod 3$, and $\xi\left(g_{0}\right)=0$ otherwise. To check that the functional 2.4) agrees on 2.2 and 2.3 , one needs

$$
\begin{aligned}
& a d-b c+\frac{1}{3}\left((a+c)^{2}+(a+c)(b+d)+(b+d)^{2}\right)= \\
& c a+a d+b d+\frac{1}{3}\left((c-a-b)^{2}+(c-a-b)(a+d)+(a+d)^{2}\right),
\end{aligned}
$$

which is indeed the case. 
2.1. Twisting cyclic cocycles. Let us assume that $x$ is of finite order, so that we always have $\xi$ satisfying 2.1 and the trace $\tau_{\omega^{t}}^{x}$ as above on $\mathbb{C}_{\omega^{t}}[G]$. Then, if $\tau^{\prime}$ is a trace on $A, \tau^{\prime} \otimes \tau_{\omega^{t}}^{x}$ is a trace on $A \otimes \mathbb{C}_{\omega^{t}}[G]$.

Let $A$ be a $G$-graded algebra, and $\left(\Omega^{*}, A \rightarrow \Omega^{0}, \tilde{\tau}: \Omega^{n} \rightarrow \mathbb{C}\right)$ be a closed graded trace model for some cyclic cocycle on $A$. Suppose that $\Omega^{*}$ is also graded over $G$, and that the homomorphism respects the grading (this is the case for the universal model $\Omega^{*}(A)$ given by $\Omega^{k}(A)=\overline{\mathrm{C}}_{k}(A)$ ). Then the twisting of $\Omega^{*}$ by $\omega$ is a subalgebra of $\Omega^{*} \otimes \mathbb{C}_{\omega^{t}}[G]$ by the inclusion of the form $[1.5)$. Thus we can restrict the closed graded trace $\tilde{\tau} \otimes \tau_{\omega^{t}}^{x}$ to $\Omega_{\omega^{t}}^{*}$, call it $\tilde{\tau}_{\omega^{t}}$. Since we have an induced homomorphism $A_{\omega^{t}} \rightarrow \Omega_{\omega^{t}}^{0}$, we obtain a closed graded trace model

$$
\left(\Omega_{\omega^{t}}^{*}, A_{\omega^{t}} \rightarrow \Omega_{\omega^{t}}^{0}, \tilde{\tau}_{\omega^{t}}: \Omega_{\omega^{t}}^{n} \rightarrow \mathbb{C}\right) .
$$

This way we can define a map (which depends on the choice of $\xi$ ) from the set of cyclic $n$-cocycles on $A$ to those on $A_{\omega^{t}}$.

Proposition 2.5. Let $x$ be an element of finite order in $G$, and $\phi\left(a_{0}, \cdots, a_{n}\right)$ be a cyclic cocycle on $A$, supported on the conjugacy class of $x$. If we denote a corresponding closed graded trace corresponding to $\phi$ by $\tilde{\tau}$, the cyclic cocycle on $A_{\omega^{t}}$ corresponding to $\tilde{\tau} \otimes \tau_{\omega^{t}}^{x}$ is given by

$$
\phi^{(t)}\left(a_{0}, \ldots, a_{n}\right)=\omega^{t}\left(g_{0}, g_{1}\right) \cdots \omega^{t}\left(g_{0} \cdots g_{n-1}, g_{n}\right) e^{t \xi\left(g_{0} \cdots g_{n}\right)} \phi\left(a_{0}, \ldots, a_{n}\right)
$$

when $a_{i}$ are homogeneous elements with $a_{i} \in A_{g_{i}}, g_{0} \cdots g_{n} \in \operatorname{Ad}_{G}(x)$.

Proof. The correspondence of $\phi$ and $\tilde{\tau}$ is given by

$$
\phi\left(a_{0}, \ldots, a_{n}\right)=\tilde{\tau}\left(a_{0} d a_{1} \cdots d a_{n}\right) .
$$

The $n$-form in $\Omega\left(A_{\omega^{t}}\right)$ can be computed as

$$
a_{0}^{\left(\omega^{t}\right)} d a_{1}^{\left(\omega^{t}\right)} \cdots d a_{n}^{\left(\omega^{t}\right)}=\omega^{t}\left(g_{0}, g_{1}\right) \cdots \omega^{t}\left(g_{0} \cdots g_{n-1}, g_{n}\right)\left(a_{0} d a_{1} \cdots d a_{n}\right)^{\left(\omega^{t}\right)}
$$

for homogeneous elements $a_{i} \in A_{g_{i}}$. Under the coaction by $\mathbb{C}[G]$, the element $\left(a_{0} d a_{1} \cdots d a_{n}\right)^{(\omega)}$ is mapped to $\left(a_{0} d a_{1} \cdots d a_{n}\right)^{(\omega)} \otimes g_{0} \cdots g_{n}$, hence $\tilde{\tau} \otimes \tau_{\omega^{t}}^{x}$ gives the formula in the right hand side of the assertion.

The inverse cocycle $\omega^{-t}(g, h)=e^{-t \omega_{0}(g, h)}$ satisfies $\left(A_{\omega^{t}}\right)_{\omega^{-t}}=A$. The associated map of cyclic cocycles for $-\xi$ is the inverse map for the above. It follows that $\phi \mapsto \phi^{(t)}$ is a linear isomorphisms from $\mathrm{HC}^{n}(A)_{x}$ to $\mathrm{HC}^{n}\left(A_{\omega^{t}}\right)_{x}$

\section{Monodromy of Gauss-Manin connection}

Let $\omega_{0}$ be a $\mathbb{C}$-valued normalized 2-cocycle on $G$. When $A$ is a $G$-graded algebra and $D \in \mathrm{C}^{*}(A, A)$, the operators $\mathbf{b}_{D}$ and $\mathbf{B}_{D}$ act on $\mathrm{C}^{*}\left(A_{\omega^{t}}\right)$ (which is equal to $\mathrm{C}^{*}(A)$ as a linear space) by transpose maps. Our goal is to compute the monodromy of the Gauss-Manin connection on the spaces $\operatorname{HP}^{*}\left(A_{\omega^{t}}\right)_{x}$ for finite order elements $x \in G$.

3.1. Finite centralizer. Suppose first that the centralizer $C_{G}(x)$ is finite. Following Section 1.3 , we put

$$
\psi(g)=\frac{1}{\left|C_{G}(x)\right|} \sum_{h \in C_{G}(x)} \omega_{0}(h, g) \quad\left(g \in C_{G}(x)\right),
$$

so that we have $d \psi(g, h)=\omega_{0}(g, h)$ on $C_{G}(x) \times C_{G}(x)$.

In order to analyze traces on the deformed algebras, we want an explicit presentation of $\xi$ on (the linear span of $\operatorname{Ad}_{G}(x)$ satisfying $-\xi(b(g \otimes h))=\omega_{0}^{a}(g, h)$. As we explained in Proposition 2.3 .

$$
-\omega_{0}^{a} h_{0}\left(g_{0}\right)=\omega_{0}^{a}\left(g^{g_{0}} g_{0},\left(g^{g_{0}}\right)^{-1}\right)
$$

is one candidate. For a reason which will become clear soon, we instead take

$$
\xi\left(g_{0}\right)= \begin{cases}\psi(x)+\omega_{0}^{a}\left(g^{g_{0}} g_{0},\left(g^{g_{0}}\right)^{-1}\right) & \left(g_{0} \in \operatorname{Ad}_{G}(x)\right) \\ 0 & \text { otherwise. }\end{cases}
$$

Note that adding a scalar to $\xi$ does not change $\xi(b(g \otimes h))$, while $e^{t \xi} \tau^{x}$ is affected. We then put

$$
\theta\left(g_{0}, g_{1}\right)= \begin{cases}\psi\left(g^{y_{1}} g_{1}\left(g^{y_{0}}\right)^{-1}\right)+\omega_{0}\left(g_{1},\left(g^{y_{0}}\right)^{-1}\right)-\omega_{0}\left(\left(g^{y_{1}}\right)^{-1}, g^{y_{1}} g_{1}\left(g^{y_{0}}\right)^{-1}\right) & \left(g_{0} g_{1} \in \operatorname{Ad}_{G}(x)\right) \\ 0 & \text { otherwise }\end{cases}
$$


with $y_{0}=g_{0} g_{1}$ and $y_{1}=g_{1} g_{0}$, as in Section 1.4. If we extend $\theta$ to $\mathbb{C}[G]^{\otimes 2}=\mathbb{C}\left[G^{2}\right]$ by linearity, it is a reduced Hochschild cochain by the normalization condition on $\omega_{0}$.

Lemma 3.1. We have $B^{\#} \theta=\xi$ as a functional on $\mathbb{C}[G]$.

Proof. As the reduced model gives $B(g)=1 \otimes g$ for $g \in G$, we have

$$
\theta\left(B\left(g_{0}\right)\right)=\psi(x)+\omega_{0}\left(g_{0},\left(g^{g_{0}}\right)^{-1}\right)-\omega_{0}\left(\left(g^{g_{0}}\right)^{-1}, x\right)
$$

for $g_{0} \in \operatorname{Ad}_{G}(x)$. Using $x=\left(g^{g_{0}} g_{0}\right)\left(g^{g_{0}}\right)^{-1}$ and the 2-cocycle identity, we indeed obtain $\xi\left(g_{0}\right)$.

Let $\omega_{0}(g, h) g h$ denote the Hochschild 2-cochain given by the linear extension of $g \otimes h \mapsto \omega_{0}(g, h) g h$.

Lemma 3.2. We have $b^{\#} \theta=\mathbf{b}_{\omega_{0}(g, h) g h}^{\#} \tau^{x}$ as a functional on $\mathbb{C}\left[G^{3}\right]$, where

$$
\mathbf{b}_{\omega_{0}(g, h) g h}^{\#} \tau^{x}\left(g_{0}, g_{1}, g_{2}\right)=\omega_{0}\left(g_{1}, g_{2}\right) \delta_{\operatorname{Ad}_{G}(x)}\left(g_{0} g_{1} g_{2}\right) \text {. }
$$

Proof. Let us keep the notation $y_{i}$ from the proof of Proposition 1.7. Expanding the definitions, we have

$$
\begin{aligned}
& \theta b\left(g_{0} \otimes g_{1} \otimes g_{2}\right)=\psi\left(g^{y_{2}} g_{2}\left(g^{y_{0}}\right)^{-1}\right)-\psi\left(g^{y_{1}} g_{1} g_{2}\left(g^{y_{0}}\right)^{-1}\right)+\psi\left(g^{y_{1}} y_{1}\left(g^{y_{2}}\right)^{-1}\right) \\
& +\omega_{0}\left(g_{2},\left(g^{y_{0}}\right)^{-1}\right)-\omega_{0}\left(\left(g^{y_{2}}\right)^{-1}, g^{y_{2}} g_{2}\left(g^{y_{2}}\right)^{-1}\right)-\omega_{0}\left(g_{1} g_{2},\left(g^{y_{0}}\right)^{-1}\right) \\
& +\omega_{0}\left(\left(g^{y_{1}}\right)^{-1}, g^{y_{1}} g_{1} g_{2}\left(g^{y_{0}}\right)^{-1}\right)+\omega_{0}\left(g_{1},\left(g^{y_{2}}\right)^{-1}\right)-\omega_{0}\left(\left(g^{y_{1}}\right)^{-1}, g^{y_{1}} g_{1}\left(g^{y_{2}}\right)^{-1}\right) \text {. }
\end{aligned}
$$

First, (by Proposition 1.6) the first three terms involving $\psi$ are equal to

$$
d \psi\left(g^{y_{1}} g_{1}\left(g^{y_{2}}\right)^{-1}, g^{y_{2}} g_{2}\left(g^{y_{0}}\right)^{-1}\right)=\omega_{0}\left(g^{y_{1}} g_{1}\left(g^{y_{2}}\right)^{-1}, g^{y_{2}} g_{2}\left(g^{y_{0}}\right)^{-1}\right) .
$$

Combining this with $\omega_{0}\left(\left(g^{y_{1}}\right)^{-1}, g^{y_{1}} g_{1} g_{2}\left(g^{y_{0}}\right)^{-1}\right)$ in $(3.3)$, we obtain

$$
\omega_{0}\left(\left(g^{y_{1}}\right)^{-1}, g^{y_{1}} g_{1}\left(g^{y_{2}}\right)^{-1}\right)+\omega_{0}\left(g_{1}\left(g^{y_{2}}\right)^{-1}, g^{y_{2}} g_{2}\left(g^{y_{0}}\right)^{-1}\right) .
$$

Note that the first term of this cancels with the last term of 3.3$)$. On the other hand, $\omega_{0}\left(g_{2},\left(g^{y_{0}}\right)^{-1}\right)-$ $\omega_{0}\left(\left(g^{y_{2}}\right)^{-1}, g^{y_{2}} g_{2}\left(g^{y_{2}}\right)^{-1}\right)$ in $(3.3)$ is equal to

$$
\omega_{0}\left(g^{y_{2}} g_{2},\left(g^{y_{0}}\right)^{-1}\right)-\omega_{0}\left(\left(g^{y_{2}}\right)^{-1}, g^{y_{2}} g_{2}\right) .
$$

Combining $\omega_{0}\left(g_{1},\left(g^{y_{2}}\right)^{-1}\right)$ in (3.3) and the second term of (3.5), we obtain

$$
\omega_{0}\left(\left(g^{y_{2}}\right)^{-1}, g^{y_{2}} g_{2}\left(g^{y_{0}}\right)^{-1}\right)+\omega_{0}\left(g_{1}, g_{2}\left(g^{y_{0}}\right)^{-1}\right) .
$$

Then the contribution from (3.6) and the first term of (3.7) is equal to $\left.\omega_{0}\left(g_{2},\left(g^{y_{0}}\right)^{-1}\right)\right)$. Combining this with $-\omega_{0}\left(g_{1} g_{2},\left(g^{y_{0}}\right)^{-1}\right)$ in $(3.3)$ and the second term of (3.7), we indeed obtain the factor $\omega_{0}\left(g_{1}, g_{2}\right)$ by the cocycle identity.

Let us consider the family of product structures $\mu_{t}(g, h)=e^{t \omega_{0}(g, h)} g h$ on $\mathbb{C}[G]$. We denote by $\omega_{0}(g, h) g h$ the 2 -cochain on $\mathbb{C}[G]$ characterized by $D(g, h)=\omega_{0}(g, h) g h$.

Proposition 3.3. We have $-\xi+\iota_{\omega_{0}(g, h) g h}^{\#} \tau^{x}=(b-B)^{\#} \theta$.

Proof. Then Lemma 3.2 says that the Hochschild cochain $D=\partial_{t} \mu_{t}$ satisfies $\mathbf{b}_{D}^{\#} \tau^{x}=b^{\#} \theta$. By the degree reason we have $\mathbf{B}_{D}^{\#} \tau^{x}=0$, so we also have $\iota_{D}^{\#} \tau^{x}=b^{\#} \theta$. Combining this with Lemma 3.1. we obtain the assertion.

Let $A$ be a $G$-graded algebra, and let us consider the smooth section algebra $\Gamma^{\infty}\left(I ; A_{\omega^{*}}\right)$ as in Section 1.1. together with the evaluation homomorphisms $\mathrm{ev}_{t}: \Gamma^{\infty}\left(I ; A_{\omega^{*}}\right) \rightarrow A_{\omega^{t}}$.

Theorem 3.4. Let $x$ be an element of $G$ with finite centralizer. Suppose that $A$ is a $G$-graded algebra, $\phi$ is a cyclic cocycle on $A$ supported on the conjugacy class of $x$, and let $\phi^{(t)}$ denote the induced cyclic cocycle on $A_{\omega^{t}}$ corresponding to $\xi$ in (3.1). When $c$ is a $(b-B)$-cycle in $\overline{\mathrm{C}}_{*}\left(\Gamma^{\infty}\left(I ; A_{\omega^{*}}\right)\right)$, the pairing $\left\langle\phi^{(t)}, \mathrm{ev}_{t} c\right\rangle$ is independent of $t$.

Proof. We want to show that the time derivative of $\left\langle\phi^{(t)}, \mathrm{ev}_{t} c\right\rangle$ is zero. Since the deformations $A \rightsquigarrow A_{\omega^{t}}$ and $\phi \rightsquigarrow \phi^{(t)}$ are additive in $t$, it is enough to prove this at $t=0$. Note also that the embedding (1.5) can be lifted to

$$
\Gamma^{\infty}\left(I ; A_{\omega^{*}}\right) \rightarrow A \otimes \Gamma^{\infty}\left(I ; \mathbb{C}_{\omega^{*}}[G]\right)
$$

Thus, we may replace $A_{\omega^{t}}$ by $A \otimes \mathbb{C}_{\omega^{t}}[G]$ and $\phi^{(t)}$ by $\phi \cup \tau_{\omega^{t}}^{x}$. Moreover, up to the shuffle product (which is a quasi-isomorphism) $\phi \cup \tau_{\omega^{t}}^{x}$ corresponds to $\phi \otimes \tau_{\omega^{t}}^{x}$ on the mixed complex $\mathrm{C}_{*}(A) \otimes \mathrm{C}_{*}\left(\mathbb{C}_{\omega^{t}}[G]\right)$. Hence it is enough to show that, if $c$ is a cycle in $\mathrm{C}_{*}(A) \otimes \mathrm{C}_{*}\left(\Gamma^{\infty}\left(I ; \mathbb{C}_{\omega^{*}}[G]\right)\right)$, the pairing

$$
\left\langle\left(\mathrm{id} \otimes \mathrm{ev}_{t}\right)(c), \phi \otimes \tau_{\omega^{t}}^{x}\right\rangle
$$


has zero derivative at $t=0$. This amounts to showing

$$
\left\langle\mathrm{ev}_{0}\left(\left(\mathrm{id} \otimes \partial_{t}\right) c\right), \phi \otimes \tau^{x}\right\rangle+\left\langle\mathrm{ev}_{0} c, \phi \otimes \xi\right\rangle=0,
$$

where we identify $\xi$ with a Hochschild 0-cochain supported on $\operatorname{Ad}_{G}(x)$.

Now, the Cartan homotopy formula 1.2 for the cochain $\partial_{t}$ implies

$$
\left(\mathrm{id} \otimes \partial_{t}\right) c=-\left(\mathrm{id} \otimes \iota_{\omega_{0}(g, h) g h}\right) c+\left(\mathrm{id} \otimes\left[b-B, \iota_{\partial_{t}}\right]\right) c .
$$

We have id $\otimes\left[b-B, \iota_{\partial_{t}}\right]=\left[b-B\right.$, id $\left.\otimes \iota_{\partial_{t}}\right]$, hence the left hand side of 3.8 is equal to

$$
-\left\langle(\mathrm{id} \otimes(b-B)) \mathrm{ev}_{0} c, \phi \otimes \theta\right\rangle
$$

by Proposition 3.3 Since $\phi$ is a $(b-B)^{\#-c o c y c l e, ~ t h i s ~ i s ~ e q u a l ~ t o ~}\left\langle(b-B) \mathrm{ev}_{0} c, \phi \otimes \theta\right\rangle=0$. This shows $\partial_{t}\left\langle\phi^{(t)}, \mathrm{ev}_{t} c\right\rangle=0$ at $t=0$ as required.

3.2. General case. Let us now turn to the general case. If $x$ is a torsion element, as remarked before Proposition 1.12. $\mathrm{H}^{n}\left(N_{G}(x) ; \mathbb{C}\right)$ is naturally isomorphic to $\mathrm{H}^{n}\left(C_{G}(x) ; \mathbb{C}\right)$. Combined with Proposition 1.3, there is a normalized 2-cocycle $\tilde{\omega}_{0}$ on $N_{G}(x)$ which is cohomologous to $\omega_{0}$ when pulled back to $C_{G}(x)$. Let $\psi$ denote a function on $C_{G}(x)$ satisfying

$$
\omega_{0}(g, h)-\psi(g)-\psi(h)+\psi(g h)=\tilde{\omega}_{0}(g, h) \quad\left(g, h \in C_{G}(x)\right) .
$$

On $\langle x\rangle$, it can be taken as

$$
\psi\left(x^{k}\right)=\frac{1}{\operatorname{ord} x} \sum_{n=0}^{\operatorname{ord} x-1} \omega_{0}\left(x^{k}, x^{n} g\right),
$$

which is independent of $g \in G$ (use the 2-cocycle condition for $x^{k}, x^{n}$, and $g$ ). Following the previous section, we define $\xi \in \mathrm{C}^{0}(\mathbb{C}[G])_{x}$ and $\theta \in \mathrm{C}^{1}(\mathbb{C}[G])_{x}$ by the same formulas (3.1) and (3.2). Lemma 3.1 holds without change, and Lemma 3.2 becomes the following.

Lemma 3.5. We have the equality $b^{\#} \theta=\mathbf{b}_{\omega_{0}(g, h) g h}^{\#} \tau^{x}+\tilde{\omega}_{0} \Xi$ as functionals on $\mathbb{C}\left[G^{3}\right]$.

Proof. The only difference from the proof of Lemma 3.2 is (3.4), which needs to be replaced by

$$
d \psi\left(g^{y_{1}} g_{1}\left(g^{y_{2}}\right)^{-1}, g^{y_{2}} g_{2}\left(g^{y_{0}}\right)^{-1}\right)=\omega_{0}\left(g^{y_{1}} g_{1}\left(g^{y_{2}}\right)^{-1}, g^{y_{2}} g_{2}\left(g^{y_{0}}\right)^{-1}\right)-\tilde{\omega}_{0}\left(g^{y_{1}} g_{1}\left(g^{y_{2}}\right)^{-1}, g^{y_{2}} g_{2}\left(g^{y_{0}}\right)^{-1}\right) .
$$

This gives the extra term $\tilde{\omega}_{0} \Xi\left(g_{0}, g_{1}, g_{2}\right)$.

Consequently, the monodromy of the Gauss-Manin connection becomes the following.

Proposition 3.6. We have $-\xi+\iota_{\omega_{0}(g, h) g h}^{\#} \tau^{x}+\tau^{x} \triangleleft \omega_{0}=(b-B)^{\#} \theta$ up to a $(b-B)$-coboundary.

Proof. The cocycle $\tilde{\omega}_{0} \Xi$ represents the image of $\left[\tilde{\omega}_{0}\right] \in \mathrm{H}^{2}\left(C_{G}(x) ; \mathbb{C}\right)$. By the choice of $\tilde{\omega}_{0}$, this is equal to the image of $\left[\omega_{0}\right]$. Since $\tau^{x}$ represents the multiplicative unit of periodic cyclic cohomology for the summand corresponding to $\mathrm{H}^{2}\left(C_{G}(x) ; \mathbb{C}\right)$, we can formally write the image of $\left[\omega_{0}\right]$ as $\tau^{x} \triangleleft \omega_{0}$.

The analogue of Theorem 3.4 is the following. Note that the assumption on $\exp \left(t\left[\omega_{0}\right]\right)$ is automatically satisfied if $\mathrm{H}^{*}\left(C_{G}(x) ; \mathbb{C}\right)$ is bounded in degree, which holds if $G$ is in the class $\mathcal{C}$ (see Lemma 1.11).

Theorem 3.7. Let $x$ be an element of finite order in $G$. Suppose that $A$ is a G-graded algebra, $\phi$ is a cyclic cocycle on $A$ supported on the conjugacy class of $x$, and let $\phi^{(t)}$ denote the induced cyclic cocycle on $A_{\omega^{t}}$ constructed with $\xi$ as above. Let us also suppose that $\exp \left(t\left[\omega_{0}\right]\right)$ makes sense in $\mathrm{H}^{*}\left(C_{G}(x) ; \mathbb{C}\right)$. When $c$ is a $(b-B)$-cocycle in $\overline{\mathrm{C}}_{*}\left(\Gamma^{\infty}\left(I ; A_{\omega^{*}}\right)\right)$, the pairing $\left\langle\phi^{(t)} \triangleleft \exp \left(-t\left[\omega_{0}\right]\right), \mathrm{ev}_{t} c\right\rangle$ is independent of $t$.

Proof. The proof is parallel to that of Theorem 3.4. but this time we want to check

$$
\left\langle\mathrm{ev}_{0}\left(\left(\mathrm{id} \otimes \partial_{t}\right) c\right), \phi \otimes \tau^{x}\right\rangle+\left\langle\mathrm{ev}_{0} c, \phi \otimes \xi\right\rangle-\left\langle\operatorname{ev}_{0} c, \phi \otimes \tau^{x} \triangleleft \omega_{0}\right\rangle=0 .
$$

The extra term $\tau^{x} \triangleleft \omega_{0}=\tau^{x} \cup \omega_{0}$ exactly corresponds to the one in Proposition 3.6 .

Corollary 3.8. With the same assumption as above, we have

$$
\left\langle\phi \triangleleft \exp \left(t\left[\omega_{0}\right]\right), \mathrm{ev}_{0} c\right\rangle=\left\langle\phi^{(t)}, \mathrm{ev}_{t} c\right\rangle .
$$

Proof. By the theorem, $\left\langle\phi^{(s)} \triangleleft \exp \left((t-s)\left[\omega_{0}\right]\right), \mathrm{ev}_{s} c\right\rangle$ is constant in $s$. At $s=0$ we obatin the left hand side of the claim, while at $s=t$ we obtain the right hand side. 


\section{Analytic PRELiminaries}

4.1. Fréchet algebras. A Fréchet algebra is given by a (possibly nonunital) $\mathbb{C}$-algebra $A$ and a sequence of (semi)norms $\|a\|_{m}(a \in A)$ for $m=1,2,3, \ldots$, such that $A$ is complete with respect to the locally convex topology defined by the $\|a\|_{m}$, and that the product map $A \times A \rightarrow A$ is (jointly) continuous. Replacing $\|a\|_{m}$ with $\sum_{k \leq m}\|a\|_{k}$ if necessary, we may and do assume that the seminorms are increasing $\left(\|a\|_{m} \leq\|a\|_{m+1}\right)$. An important condition one may ask on these seminorms is the m-convexity: each $\|a\|_{m}$ is submultiplicative, that is $\|a b\|_{m} \leq\|a\|_{m}\|b\|_{m}$. If we can characterize the topology of $A$ by seminorms satisfying this condition, $A$ is then called an $m$-algebra.

Example 4.1. By $C^{1}(I)$ we mean the space of continuous functions $f$ on $I=[0,1]$ which admit continuous derivative $\partial_{t} f$, where at the endpoints we consider one sided deriatives

$$
\left(\partial_{t} f\right)(0)=\lim _{t \rightarrow+0} \frac{f(t)-f(0)}{t}, \quad\left(\partial_{t} f\right)(1)=\lim _{t \rightarrow+0} \frac{f(1)-f(1-t)}{t} .
$$

The spaces $C^{k}(I)$ for $k=2,3, \ldots$ are defined similarly in an inductive way. Each of these is a Banach algebra, with respect to the submultiplicative norm

$$
\|f\|_{k}=\sum_{j=0}^{k} \frac{1}{j !}\left\|\partial_{t}^{j} f\right\|
$$

with $\|f\|=\max _{0 \leq t \leq 1}|f(t)|$. Their intersection $C^{\infty}(I)=\bigcap_{k=0}^{\infty} C^{k}(I)$ is the space of smooth functions on $I$, which is an $m$-algebra topologized by the above norms.

4.2. Tensor products. Let $\mathcal{A}$ and $\mathcal{A}^{\prime}$ be Fréchet spaces, respectively with seminorms $\left(\|y\|_{m}\right)_{m=1}^{\infty}$ and $\left(\|z\|_{n}^{\prime}\right)_{n=1}^{\infty}$. The projective tensor product $\mathcal{A} \hat{\otimes} \mathcal{A}^{\prime}$ is the completion of algebraic tensor product $\mathcal{A} \otimes \mathcal{A}^{\prime}$ with respect to the seminorms

$$
\|x\|_{(m, n)}=\inf \left\{\sum_{i=1}^{k}\left\|y_{i}\right\|_{m}\left\|z_{i}\right\|_{n} \mid k \in \mathbb{N}, x=\sum_{i=1}^{k} y_{i} \otimes z_{i}\right\} .
$$

When $\mathcal{A}$ and $\mathcal{A}^{\prime}$ are $m$-algebras, $\mathcal{A} \hat{\otimes} \mathcal{A}^{\prime}$ is again an $m$-algebra (note that tensor product of submultiplicative seminorms are again submultiplicative).

Example 4.2. When $A$ is a Banach space, $\ell_{1}(G) \hat{\otimes} A$ is identified with the space $\ell_{1}(G ; A)$ of absolutely summable functions $f: G \rightarrow A$, that is, $\sum_{g} \delta_{g} \otimes f(g)$ represents an element of $\ell_{1}(G) \hat{\otimes} A$ if and only if $\|f\|_{1}=\sum_{g}\|f(g)\|$ is finite.

When $\mathcal{A}$ is a Fréchet space, the space $C^{\infty}(I ; \mathcal{A})$ of $\mathcal{A}$-valued smooth functions on $I$ is identified with the injective tensor product (or $\epsilon$-tensor product) $C^{\infty}(I) \hat{\otimes}_{\epsilon} \mathcal{A}$ of $C^{\infty}(I)$ and $\mathcal{A}$, cf. 36. Theorem 2.3; 47, Chapter 44]. Since $C^{\infty}(I)$ is a nuclear Fréchet space, (see, e.g. [32]), the topology of this space is the same as that of the projective tensor product. We will freely use these different interpretations.

4.3. Banach KK-groups. Let us briefly review the construction of the group $K K^{\mathrm{Ban}}(A, B)$ for Banach algebras $A$ and $B$, see 23,50 for details.

The basic ingredient is a $B$-pair, which is a Banach algebra analogue of right Hilbert $\mathrm{C}^{*}$-modules. Let $E^{\langle}, E^{\rangle}$be Banach spaces endowed with submultiplicative actions of $B$, from left on $E^{\zeta}$ and from right on $E^{\rangle}$. Then, a $B$-pair $E$ is given by such spaces endowed with a $B$-valued pairing

$$
E^{\langle} \times E^{\rangle} \rightarrow B, \quad(x, y) \mapsto\langle x, y\rangle_{B},
$$

satisfying $\langle b x, y\rangle_{B}=b\langle x, y\rangle_{B},\langle x, y b\rangle_{B}=\langle x, y\rangle_{B} b$, and $\left\|\langle x, y\rangle_{B}\right\| \leq\|x\| \cdot\|y\|$. When $E_{0}$ and $E_{1}$ are such $B$-pairs, a morphism of $B$-pairs $T: E_{0} \rightarrow E_{1}$ is given by module maps $T^{\curlywedge}: E_{1}^{\langle} \rightarrow E_{0}^{\curlywedge}$ and $T^{\rangle}: E_{0}^{\rangle} \rightarrow E_{1}^{\rangle}$ satisfying an analogue of the adjointability condition for maps between Hilbert modules. One also has an analogue of compact morphisms for Hilbert modules.

Then, elements of $K K^{\mathrm{Ban}}(A, B)$ are represented by the data $\left(E_{*}, \pi, T\right)$, where $E_{*}=E_{0} \oplus E_{1}$ is a $\mathbb{Z} / 2 \mathbb{Z}$ graded $B$-pair, $\pi$ is a representation of $A$ as even endomorphisms of $E_{*}$, and $T$ is an odd endomorphism such that $\pi(a)\left(\mathrm{id}-T^{2}\right)$ and $[T, \pi(a)]$ for all $a \in A$. Such data define maps $K_{i}(A) \rightarrow K_{i}(B)$ for $i=0,1$, analogous to the usual KK-theory.

When $A$ and $B$ admit isometric actions of $G$ and $L$ is a length function on $G$, we also have $K K_{G, L}^{\mathrm{Ban}}(A, B)$ generated by an equivariant version of the above data with the failure of $G \curvearrowright E$ being isometric controlled by $L$. While this generalization is important for the Baum-Connes conjecture of $G$, it appears only indirectly in the following section. 
4.4. Analytic models of twisting. When $\omega$ is a $\mathbb{T}$-valued normalized 2-cocycle on $G$, we can represent $\mathbb{C}_{\omega}[G]$ on $\ell_{2}(G)$ by bounded operators, as

$$
\left(\lambda_{g}^{(\omega)} f\right)(h)=\omega\left(g, g^{-1} h\right) f\left(g^{-1} h\right) \quad\left(f \in \ell_{2}(G)\right),
$$

called the $\omega$-twisted left regular representation. The reduced twisted group $C^{*}$-algebra $C_{r, \omega}^{*}(G)=C_{r}^{*}(G, \omega)$ is defined as the norm closure of $\mathbb{C}_{\omega}[G]$ inside $B\left(\ell_{2}(G)\right)$.

When $A$ is a $G$-C ${ }^{*}$-algebra, the Banach space

$$
\ell_{1}(G ; A)=\left\{f: G \rightarrow A \mid\|f\|_{1}=\sum_{g \in G}\|f(g)\|<\infty\right\}
$$

has a structure of Banach $*$-algebra by convolution product. When $\omega$ is a normalized $\mathbb{T}$-valued cocycle, we can again twist the product of $\ell_{1}(G ; A)$ into a Banach $*$-algebra, with respect to the same involution map (recall that $\omega$ is assumed to satisfy $\omega\left(g, g^{-1}\right)=1$ ). If $G$ is amenable, $C_{r, \omega}^{*}(G)$ is the $\mathrm{C}^{*}$-enveloping algebra of $\ell_{1}(G)=\ell_{1}(G ; \mathbb{C})$ with this product.

More generally, if $A$ is the reduced section algebra of a Fell bundle over $G$ (that is, a $\mathrm{C}^{*}$-algebra with injective coaction $A \rightarrow A \otimes C_{r}^{*}(G)$ ), we can define a $\mathrm{C}^{*}$-algebraic model of $A_{\omega}$ on the right Hilbert $A_{e}$-module $L^{2}(A)_{A_{e}}$, so that $A_{\omega}$ is again the reduced section algebra of another Fell bundle [53].

\section{SMOOTh MODELS}

We want to consider Fréchet variants of $\Gamma^{\infty}(I ; \mathbb{C}[G])$ in the previous section.

5.1. $\ell_{1}$-Schwartz algebra. Let $\ell: G \rightarrow[0, \infty)$ be a length function on $G$; a function satisfying $\ell(e)=0$ and $\ell(g h) \leq \ell(g)+\ell(h)$. Let $\mathcal{A}$ be a Fréchet algebra with seminorms $\|a\|_{m}$, and let $\alpha: G \curvearrowright \mathcal{A}$ be an action of $G$ which is $\ell$-tempered 46 :

$$
\forall m \exists C, k, n:\left\|\alpha_{g}(x)\right\|_{m} \leq C(\ell(g)+1)^{k}\|x\|_{n} .
$$

We then put

$$
S_{1}(G ; \mathcal{A})=\left\{f: G \rightarrow \mathcal{A} \mid \forall k, m: \sum_{g}(\ell(g)+1)^{k}\|f(g)\|_{m}<\infty\right\} .
$$

As a Fréchet space this is just the projective tensor product of $S_{1}(G)=S_{1}(G ; \mathbb{C})$ and $\mathcal{A}$. Concretely, the seminorms

$$
\|f\|_{d, m}=\sum_{g}(\ell(g)+1)^{d}\|f\|_{m}
$$

topologize $S_{1}(G ; \mathcal{A})$, and since $\|f\|_{m}$ is increasing in $m$, the ones $\|f\|_{m}^{\prime}=\|f\|_{m, m}$ also topologize $S_{1}(G ; \mathcal{A})$. With respect to the crossed product algebra structure

$$
\left(f * f^{\prime}\right)(g)=\sum_{g^{\prime}} \alpha_{g^{\prime}}\left(f\left(g g^{\prime}\right)\right) f^{\prime}\left(\left(g^{\prime}\right)^{-1}\right),
$$

$S_{1}(G ; \mathcal{A})$ is an $m$-algebra if $\mathcal{A}$ is a one [45, Theorem 3.1.7]. Let us denote the twistings of $\ell_{1}(G)$ (resp. $S_{1}(G)$ ) by $\ell_{1}(G, \omega)$ (resp. $S_{1}(G, \omega)$ ). Note that the traces $\tau^{x}$ are well-defined over $\ell_{1}(G)$, hence also over $S_{1}(G)$.

Let $A$ be a $G$-C ${ }^{*}$-algebra, and consider the crossed product algebra structure $\ell_{1}(G ; A)$ defined as above. If there are Dirac-dual Dirac morphisms in $K K^{G}$ with $\gamma=1$ in $K K_{G, L}^{\mathrm{Ban}}(\mathbb{C}, \mathbb{C})$ for some $L$, the assembly map

$$
K_{*}^{G}(\underline{E} G, A) \rightarrow K_{*}\left(\ell_{1}(G ; A)\right)
$$

is an isomorphism [23. For good $G$ (including Haagerup groups 16 and word hyperbolic groups [25]), the assembly maps

$$
K_{*}^{G}(\underline{E} G, A) \rightarrow K_{*}\left(G \ltimes_{r} A\right)
$$

is also an isomorphism, with $G \ltimes_{r} A$ being the reduced crossed product $\mathrm{C}^{*}$-algebra. It follows that the natural map $K_{*}\left(\ell_{1}(G ; A)\right) \rightarrow K_{*}\left(G \ltimes_{r} A\right)$ is an isomorphism for such groups. In the rest of the section we assume that this is the case.

Let $\omega_{0}$ be a $\sqrt{-1} \mathbb{R}$-valued 2-cocycle on $G$, and consider the associated $\mathbb{T}$-valued cocycles

$$
\omega^{t}(g, h)=\exp \left(t \omega_{0}(g, h)\right) \quad(t \in I) .
$$

We then obtain a 'continuous' family of twisted group $\mathrm{C}^{*}$-algebras $C_{r, \omega^{t}}^{*}(G)$, and the $\mathrm{C}^{*}$-algebra of continuous sections $\Gamma\left(I ; C_{r, \omega^{*}}^{*}(G)\right)$ plays a central role in the comparison of their K-groups. By definition 
$\Gamma\left(I ; C_{r, \omega^{*}}^{*}(G)\right)$ is a completion of $\ell_{1}(G ; C(I))$ with respect to the representation on Hilbert $C(I)$-module $\Gamma\left(I ; \ell_{2}(G)\right)$, twisted by the $\mathcal{U}(C(I))$-valued 2-cocycle $\omega^{*}$ on $G[12$.

Recall that $C_{r, \omega}^{*}(G)$ is strongly Morita equivalent to $G \ltimes_{r} K\left(\ell_{2}(G)\right.$ ), where $G$ is acting on $K\left(\ell_{2}(G)\right.$ ) by $\left(\operatorname{Ad}_{\lambda_{g}^{(\varpi)}}\right)_{g \in G}\left[34\right.$. Concretely, the imprimitivity bimodule is given by the Hilbert $C_{r, \omega}^{*}(G)$-module $C_{r, \omega}^{*}(G) \otimes \ell_{2}(G)$, where the left action of $G \ltimes_{r} K\left(\ell_{2}(G)\right)$ is given by $\lambda_{g}^{(\omega)} \otimes \lambda_{g}^{(\bar{\omega})}$ for $g \in G$ and $1 \otimes T$ for $T \in K\left(\ell_{2}(G)\right)$.

The $\ell_{1}$-version of this is a cycle in $K K^{\mathrm{Ban}}\left(\ell_{1}\left(G ; K\left(\ell_{2}(G)\right)\right), \ell_{1}(G, \omega)\right)$, as follows. The graded $\ell_{1}(G ; \omega)$ pair $E_{*}$ is given by $E_{1}=0$ (thus $T=0$ ), while $E_{0}^{\rangle}=\ell_{1}(G) \hat{\otimes} \ell_{2}(G)$ and $E_{0}^{\langle}$is its complex conjugate $\overline{\ell_{1}(G) \hat{\otimes} \ell_{2}(G)}=\left\{\bar{x} \mid x \in \ell_{2}(G)\right.$ and $\left.E_{0}^{\langle}\right\}$. The pairing is given by

$$
\left\langle\overline{f^{1} \otimes \xi^{1}}, f^{2} \otimes \xi^{2}\right\rangle_{\ell_{1}(G ; \omega)}=\left(\xi^{2}, \xi^{1}\right)\left(f^{1}\right)^{*} *_{\omega} f^{2} .
$$

The right module structure on $E_{0}^{\rangle}$is given by $(f \otimes \xi) f^{\prime}=\left(f *_{\omega} f^{\prime}\right) \otimes \xi$, and the left module structure on $E_{0}^{\langle}$is given by $f^{\prime}(\overline{f \otimes \xi})=\overline{f *_{\omega}\left(f^{\prime}\right)^{*} \otimes \xi}$. On this $B$-pair, $\ell_{1}\left(G ; K\left(\ell_{2}(G)\right)\right)$ acts by endomorphisms

$$
\pi^{\rangle}(f)\left(f^{\prime} \otimes \xi\right)=\sum_{g} \lambda_{g}^{(\omega)} f^{\prime} \otimes \lambda^{(\bar{\omega})} f(g) \xi, \quad \pi^{\langle}(f)\left(\overline{f^{\prime} \otimes \xi}\right)=\sum_{g} \overline{\lambda_{g}^{(\omega)} f^{\prime} \otimes f\left(g^{-1}\right)^{*} \lambda^{(\bar{\omega})} \xi} .
$$

This cycle implements the isomorphism $K_{*}\left(\ell_{1}\left(G ; K\left(\ell_{2}(G)\right)\right)\right) \simeq K_{*}\left(\ell_{1}(G, \omega)\right)$.

Collecting these discussions, $\ell_{1}(G, \omega) \rightarrow C_{r, \omega}^{*}(G)$ induces isomorphisms of $K$-groups 31 .

Proposition 5.1. The evaluation map $K_{*}\left(\ell_{1}(G ; C(I))\right) \rightarrow K_{*}\left(\ell_{1}\left(G, \omega^{t}\right)\right)$ is surjective for each $t$.

Proof. We already know the corresponding statement for $\mathrm{C}^{*}$-algebras, and that $K_{*}\left(\ell_{1}\left(G, \omega^{t}\right)\right)$ is isomorphic to $K_{*}\left(C_{r, \omega^{t}}^{*}(G)\right)$. We also know that $K_{*}\left(\ell_{1}\left(G ; C(I) \otimes K\left(\ell_{2}(G)\right)\right)\right) \simeq K_{*}\left(G \ltimes_{r} C(I) \otimes K\left(\ell_{2}(G)\right)\right)$. Thus, we just need to verify that the evaluation map in the assertion factors the isomorphism

$$
K_{*}\left(\ell_{1}\left(G ; C(I) \otimes K\left(\ell_{2}(G)\right)\right)\right) \rightarrow K_{*}\left(\ell_{1}\left(G, \omega^{t}\right)\right)
$$

for each $t$.

If we carry out a similar construction for $\ell_{1}(G ; C(I))$ and $\ell_{1}\left(G ; C(I) \otimes K\left(\ell_{2}(G)\right)\right.$, the Banach space $\ell_{1}\left(G ; C\left(I ; \ell_{2}(G)\right)\right)$ gives a cycle in $K K^{\mathrm{Ban}}\left(\ell_{1}\left(G ; C(I) \otimes K\left(\ell_{2}(G)\right)\right), \ell_{1}(G ; C(I))\right)$, hence a map

$$
K_{*}\left(\ell_{1}\left(G ; C(I) \otimes K\left(\ell_{2}(G)\right)\right)\right) \rightarrow K_{*}\left(\ell_{1}(G ; C(I))\right) .
$$

This is the desired factorization.

Since the natural (semi)norms of $S_{1}\left(G ; C^{\infty}(I)\right)$ are stronger than that of $\ell_{1}(G ; C(I))$, we may regard $S_{1}\left(G ; C^{\infty}(I)\right)$ as a subspace of $\Gamma\left(I ; C_{r, \omega^{*}}^{*}(G)\right)$. In order to control the time derivatives of the product in this algebra, we need to impose the polynomial growth assumption on $\omega_{0}$. Namely, $\omega_{0}$ is of polynomial growth with respect to $\ell$ if

$$
\left|\omega_{0}(g, h)\right| \leq C(1+\ell(g))^{M}(1+\ell(h))^{M}
$$

holds for some positive constants $C$ and $M$ large enough.

Proposition 5.2 (cf. 46, Theorem 6.7]). If $\omega_{0}$ is of polynomial growth, $S_{1}\left(G ; C^{\infty}(I)\right)$ is a spectral subalgebra of $\ell_{1}(G ; C(I))$.

Proof. First, $S_{1}(G ; C(I))$ is a strongly spectral invariant subalgebra of $\ell_{1}(G ; C(I))$. On this part the argument for [46. Theorem 6.7] applies without change, since the twisting by $\omega^{t}$ does not contribute to the norm of these algebras.

We first need to verify that $S_{1}\left(G ; C^{\infty}(I)\right)$ is a subalgebra of $S_{1}(G ; C(I))$. Let $f$ and $f^{\prime}$ be elements of $S_{1}\left(G ; C^{\infty}(I)\right) \simeq C^{\infty}\left(I ; S_{1}(G)\right)$. Their product is represented by the function

$$
(t, g) \mapsto \sum_{h \in G} \omega^{t}\left(h, h^{-1} g\right) f_{t}(h) f_{t}^{\prime}\left(h^{-1} g\right) .
$$

Since $\left|\omega^{t}\left(h, h^{-1} g\right)\right|=1$, at each $t$ we indeed obtain an element of $S_{1}\left(G, \omega^{t}\right)$. We thus need to show that this function is smooth in $t$. Let us denote by $C$ and $M$ the constants controlling the growth of $\omega_{0}$ as above, and put $m_{+}=m(1+M)$.

As an illustration let us first give an estimate for the first order derivative. We have

$$
\begin{aligned}
\partial_{t}\left(f *_{\omega^{*}}\right. & \left.f^{\prime}\right)_{t}(g) \\
& =\sum_{h} \omega^{t}\left(h, h^{-1} g\right)\left(\omega_{0}\left(h, h^{-1} g\right) f_{t}(h) f_{t}^{\prime}\left(h^{-1} g\right)+\left(\partial_{t} f_{t}(h)\right) f_{t}^{\prime}\left(h^{-1} g\right)+f_{t}(h) \partial_{t} f_{t}^{\prime}\left(h^{-1} g\right)\right) .
\end{aligned}
$$


For any $k$, the second term can be estimated as $\left\|\partial_{t} f *_{\omega^{t}} f^{\prime}\right\|_{k, 0} \leq\left\|\partial_{t} f\right\|_{k, 0}\left\|\partial_{t} f^{\prime}\right\|_{k, 0}$, so

$$
g \mapsto \sum_{h} \omega^{t}\left(h, h^{-1} g\right)\left(\partial_{t} f_{t}(h)\right) f_{t}^{\prime}\left(h^{-1} g\right)
$$

is an element of $S_{1}(G)$. The third term admits a similar estimate. As for the first term, we need to estimate

$$
\sum_{g}\left(\sum_{h}\left|\omega_{0}\left(h, h^{-1} g\right)\right|\left|f_{t}(h)\right|\left|f_{t}^{\prime}\left(h^{-1} g\right)\right|\right)(1+\ell(g))^{k} .
$$

If we put $\tilde{f}_{t}(g)=f_{t}(g)(1+\ell(g))^{M}$, the above is bounded by

$$
C\left\||\tilde{f}| *\left|\tilde{f}^{\prime}\right|\right\|_{k, 0} \leq C\||\tilde{f}|\|_{k, 0}\left\|\left|\tilde{f}^{\prime}\right|\right\|_{k, 0}=C\|f\|_{k_{+}, 0}\left\|f^{\prime}\right\|_{k_{+}, 0} .
$$

This shows that $f * \omega^{*} f^{\prime}$ indeed has a bounded first order derivative.

Let us now proceed to the higher order derivatives. For $f \in S_{1}\left(G ; C^{m}(I)\right)$, put

$$
\|f\|_{m}=\sum_{k=0}^{m} \frac{1}{k !} \max _{s \in I}\left\|\partial_{t}^{k} f_{s}\right\|_{m},
$$

where on the right hand side we take the norm $\left\|f^{\prime}\right\|_{m}=\sum_{g}(1+\ell(g))^{m}\left|f^{\prime}(g)\right|$ for $f^{\prime}=\partial_{t}^{k} f_{s}$. Given $f^{1}, \ldots, f^{n} \in S_{1}\left(G ; C^{\infty}(I)\right)$, we claim that there is an estimate

$$
\left\|f^{1} *_{\omega^{*}} \cdots *_{\omega^{*}} f^{n}\right\|_{m} \leq e^{n C}\left\|f^{1}\right\|_{m_{+}} \cdots\left\|f^{n}\right\|_{m_{+}},
$$

which implies that $S_{1}\left(G ; C^{\infty}(I)\right)$ is closed under product. For $\left(g_{1}, \ldots, g_{n}\right) \in G^{n}$, put

$$
\omega_{0}^{(n)}\left(g_{1}, \ldots, g_{n}\right)=\omega_{0}\left(g_{1}, g_{2}\right)+\omega_{0}\left(g_{1} g_{2}, g_{3}\right)+\cdots+\omega_{0}\left(g_{1} \cdots g_{n-1}, g_{n}\right) .
$$

Then the product $f^{1} \cdots f^{n}$ in $\Gamma\left(I ; C_{r, \omega^{*}}^{*}(G)\right)$ is represented by

$$
h_{t}(g)=\sum_{g_{1} \cdots g_{n}=g} f^{1}\left(g_{1}\right) \cdots f^{n}\left(g_{n}\right) e^{t \omega_{0}^{(n)}\left(g_{1}, \ldots, g_{n}\right)} .
$$

Then, $\partial_{t}^{k} h_{s}(g)$ is given by

$$
\sum_{a_{1}+\cdots+a_{n+1}} \frac{k !}{a_{1} ! \cdots a_{n+1} !} \partial_{t}^{a_{1}} f_{s}^{1}\left(g_{1}\right) \cdots \partial_{t}^{a_{n}} f_{s}^{n}\left(g_{n}\right) \omega_{0}^{(n)}\left(g_{1}, \ldots, g_{n}\right)^{a_{n+1}} e^{t \omega_{0}\left(g_{1}, \ldots, g_{n}\right)} .
$$

Using

$$
\omega_{0}^{(n)}\left(g_{1}, \ldots, g_{n}\right)^{a_{n+1}}=\sum_{b_{1}+\cdots+b_{n-1}} \frac{a_{n+1} !}{b_{1} ! \cdots b_{n-1} !} \omega_{0}\left(g_{1}, g_{2}\right)^{b_{1}} \omega_{0}\left(g_{1} g_{2}, g_{3}\right)^{b_{2}} \cdots \omega_{0}\left(g_{1} \cdots g_{n-1}, g_{n}\right)^{b_{n-1}},
$$

we can bound $\frac{1}{k !} \max _{s}\left\|\partial_{t}^{k} h_{s}\right\|_{m}$ by

$$
\begin{aligned}
& \sum_{\substack{a_{1}+\cdots+a_{n}+b_{1} \\
+\cdots+b_{n-1}=k}} \frac{C^{b_{1}+\cdots+b_{n-1}}}{a_{1} ! \cdots a_{n} ! b_{1} ! \cdots b_{n-1} !} \\
& \quad \times\left\|\left(\cdots\left(\widetilde{\partial_{t}^{a_{1}} f_{s}^{1}}\left(b_{1}\right){\widetilde{\partial_{t}^{a_{2}} f_{s}^{2}}}^{\left(b_{1}\right)}\right)^{\sim\left(b_{2}\right)} \widetilde{\partial_{t}^{a_{3}} f^{3}}\left(b_{2}\right) \cdots\right)^{\sim\left(b_{n-1}\right)}{\widetilde{\partial_{t}^{a_{n}} f_{s}^{n}}}^{\left(b_{n-1}\right)}\right\|_{m},
\end{aligned}
$$

where we put $\tilde{f}^{(b)}(g)=f(g)(1+\ell(g))^{M b}$. Repeatedly using the estimates of the form (5.4), we can bound this by

$$
\begin{aligned}
\sum_{\substack{a_{1}+\cdots+a_{n}+b_{1} \\
+\cdots+b_{n-1}=k}} \frac{C^{b_{1}+\cdots+b_{n-1}}}{a_{1} ! \cdots a_{n} ! b_{1} ! \cdots b_{n-1} !}\left\|\partial_{t}^{a_{1}} f_{s}^{1}\right\|_{m+\left(b_{1}+\cdots+b_{n-1}\right) M} & \\
& \times\left\|\partial_{t}^{a_{2}} f_{s}^{2}\right\|_{m+\left(b_{1}+\cdots+b_{n-1}\right) M} \cdots\left\|\partial_{t}^{a_{n}} f_{s}^{n}\right\|_{m+b_{n-1} M}
\end{aligned}
$$

Then, using $b_{1}+\cdots+b_{n-1} \leq k \leq m$ and $\sum_{i} C^{b_{i}} / b_{i} ! \leq e^{C}$, we obtain the desired estimate (5.5).

It remains to show that $S_{1}\left(G ; C^{\infty}(I)\right)$ is closed under taking multiplicative inverses. Now, suppose that $f \in S_{1}\left(G ; C^{\infty}(I)\right)$ is invertible in $S_{1}(G ; C(I))$. The assertion follows if we can show that $f^{-1}$ 
belongs to $S_{1}\left(G ; C^{m}(I)\right)$ for all $m$. If $f \in S_{1}\left(G ; C^{m}(I)\right)$ is invertible in $S_{1}(G ; C(I))$, we fix $C_{1}<1$ and approximate $f^{-1}$ by $f^{\prime} \in S_{1}\left(G ; C^{m}(I)\right)$ satisfying

$$
\left\|f^{\prime}-f^{-1}\right\|_{m_{+}, 0}<\frac{C_{1}}{e^{3 C}\|f\|_{m_{+}, 0}}
$$

so that $x=1-f^{\prime} f$ satisfies $\|x\|_{m_{+}, 0}<C_{1} e^{-C}$. Then, for $n>m$, the above estimate for $f^{1}=\cdots=f^{n}=x$ shows

$$
\left\|x^{n}\right\|_{m, m} \leq e^{n C} n^{m}\|x\|_{m_{+}, m}^{m}\|x\|_{m_{+}, 0}^{n-m}=o\left(C_{2}^{n}\right)
$$

for any $C_{1}<C_{2}<1$. This shows the convergence of $1+x+x^{2}+\cdots$, hence $f^{\prime} f$ is invertible in $S_{1}\left(G ; C^{m}(I)\right)$.

Corollary 5.3. The evaluation map $K_{*}\left(S_{1}\left(G ; C^{\infty}(I)\right)\right) \rightarrow K_{*}\left(S_{1}\left(G, \omega^{t}\right)\right)$ is surjective for each $t$.

Proof. A similar (but simpler) argument as the above proposition shows that $S_{1}\left(G, \omega^{t}\right)$ is a spectral subalgebra of $\ell_{1}\left(G, \omega^{t}\right)$. We thus know that the horizontal arrows in

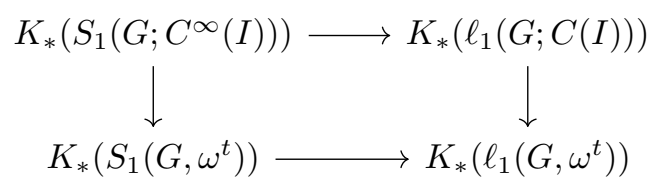

are isomorphisms. Moreover, the right vertical arrow is surjective by Proposition 5.1

Remark 5.4. When $G$ is of polynomial growth, there is possibly a more direct approach based on the following result of Schweitzer [46]: suppose that $A$ is a $G$-C $C^{*}$-algebra, and $\mathcal{A}$ be a dense $G$-invariant Fréchet subalgebra satisfying the differential seminorm condition such that the induced action is $\ell$ tempered. Then $S_{1}(G ; \mathcal{A})$ is a spectral subalgebra of $G \ltimes_{r} A$. However, this result is hopeless without polynomial growth assumption. Indeed, $\ell_{1}(G)$ is not a spectral subalgebra of $C_{r}^{*}(G)$ if $G$ contains a free semigroup of two generators 3,17 .

Remark 5.5. The subscript 1 in $S_{1}(G ; A)$ corresponds to the fact that the definition in (5.1) is in terms of the ' $\ell_{1}$ norm'. When $A$ is a pre $\mathrm{C}^{*}$-algebra, the $\ell_{2}$ version makes sense as

$$
S_{2}(G ; A)=\left\{f: G \rightarrow A \mid \forall k, m:\left\|\sum_{g} \alpha_{g}^{-1}\left(f(g)^{*} f(g)\right)(1+\ell(g))^{2 k}\right\|_{m}<\infty\right\} .
$$

This way $S_{2}(G ; \mathbb{C})$ is equal to $H_{\ell}^{\infty}(G)$, see the next section. However, the spectral invariance of $S_{2}(G ; A)$ in $G \ltimes_{r} A$ is proved only when $G$ is of polynomial growth and $A$ is a commutative $\mathrm{C}^{*}$-algebra 20], and $S_{2}\left(G ; \ell_{\infty} G\right)$ is a spectral subalgebra of $G \ltimes_{r} \ell_{\infty} G$ (if and) only if $G$ is of polynomial growth [7. Note also that we have $S_{1}(G)=S_{2}(G)$ if and only if $G$ is of polynomial growth [21]. For hyperbolic groups, there is a more elaborate choice of rapid decay functions 38$]$ which are invariant under holomorphic functional calculus on $C_{r}^{*}(G)$ and at the same time supporting many traces.

\section{2. $\ell_{2}$-Schwartz algebra for groups of rapid decay. For $s>0$, put}

$$
H_{\ell}^{s}(G)=\left\{f:\left.G \rightarrow \mathbb{C}\left|\sum_{g \in G}\right| f(g)\right|^{2}(\ell(g)+1)^{2 s}<\infty\right\},
$$

and $H_{\ell}^{\infty}(G)=\bigcap_{s>0} H_{\ell}^{s}(G)$. Recall that $G$ is said to be of rapid decay 21] if $H_{\ell}^{\infty}(G)$ is a subspace of $C_{r}^{*}(G)$ for some proper length function $\ell$. This is equivalent to $H_{\ell}^{s}(G) \subset C_{r}^{*}(G)$ for large enough $s$. Note that when $G$ is finitely generated, it is of rapid decay if and only if $H_{\ell}^{\infty}(G) \subset C_{r}^{*}(G)$ for the word length $\ell$ for some symmetric generating set. Put

$$
\|f\|_{\ell, s}=\sqrt{\sum_{g \in G}|f(g)|^{2}(\ell(g)+1)^{2 s}},
$$

so that $H_{\ell}^{s}(G)$ is the completion of $\mathbb{C}[G]$ by this norm. When $H_{\ell}^{s}(G) \subset C_{r}^{*}(G)$, there is $C>0$ such that whenever $f$ is supported on $B_{R}(e)$ (the ball around $e$ of radius $R$ ), we have $\|f\|_{C_{r}^{*}(G)} \leq C R^{s}\|f\|_{\ell, s}$, or that there is a polynomial $P(X)$ such that

$$
\|f * g\|_{C_{r}^{*}(G)} \leq P(R)\|f\|_{\ell_{2}(G)}\|g\|_{\ell_{2}(G)}
$$


whenever $f$ is supported on $B_{R}(e)$. The last condition implies $\|f * g\|_{\ell, s} \leq K(s)\|f\|_{\ell, s}\|g\|_{\ell, s}$ for $s \geq$ $\operatorname{deg} P(X)$ and

$$
K(s)=2^{s} \sqrt{P(1)^{2}+\sum_{n} \frac{P\left(2^{n}\right)^{2}}{\left(1+2^{n-1}\right)^{2 s}}}
$$

so that $H_{\ell}^{s}(G)$ is a Banach algebra with respect to the rescaled norm $\|f\|_{\ell, s}^{\prime}=K(s)\|f\|_{\ell, s}$ 24, Proposition 1.2]. Modifying the factor $K(s)$ if needed, we may assume that $\|f\|_{\ell, s}^{\prime}$ is increasing in $s$. This way $H_{\ell}^{\infty}(G)$ is an $m$-algebra. It is also closed under holomorphic functional calculus inside $C_{r}^{*}(G)$.

Let $G$ be a group of rapid decay, and $\omega$ be a normalized $\mathbb{T}$-valued 2-cocycle on $G$. As explained in 31. Proposition 6.11], $H_{\ell}^{\infty}(G)$ is also a subalgebra of $C_{r, \omega}^{*}(G)$ thanks to the estimate

$$
\left\|f *_{\omega} g\right\|_{\ell, s} \leq\||f| *|g|\|_{\ell, s} \leq K(s)\|f\|_{\ell, s}\|g\|_{\ell, s}
$$

for large enough $s$. Let us denote this algebra by $H_{\ell}^{\infty}(G, \omega)$. It is again invariant under holomorphic functional calculus in $C_{r, \omega}^{*}(G)$.

The smooth section algebra $S_{1}\left(G ; C^{\infty}(I)\right)$ in the previous section needs to be replaced by $C^{\infty}\left(I ; H_{\ell}^{\infty}(G)\right)$. Then, an analogue of the first half of Proposition 5.2 holds.

Proposition 5.6. Suppose that $\omega_{0}$ is of polynomial growth. Then $C^{\infty}\left(I ; H_{\ell}^{\infty}(G)\right)$ is an m-convex subalgebra of $\Gamma\left(I ; C_{r, \omega^{*}}^{*}(G)\right)$.

Proof. Let $f$ and $f^{\prime}$ be elements of $C^{\infty}\left(I ; H_{\ell}^{\infty}(G)\right)$. Their product in $\Gamma\left(I ; C_{r, \omega^{*}}^{*}(G)\right)$ is represented by 5.2. and by the estimate 5.7), at each $t$ we indeed obtain an element of $H_{\ell}^{\infty}\left(G, \omega^{t}\right)$. We thus need to show that this function is smooth in $t$.

The proof is completely analogous to the first half of Proposition 5.2, with slight modifications using (5.7). For example, in (5.3) the second term can be estimated as

$$
\left\|\partial_{t} f *_{\omega^{t}} f^{\prime}\right\|_{\ell, s} \leq K(s)\left\|\partial_{t} f\right\|_{\ell, s}\left\|\partial_{t} f^{\prime}\right\|_{\ell, s}
$$

as in (5.7), and the third term admits a similar estimate. As for the first term, we need to estimate

$$
\sum_{g}\left(\sum_{h}\left|\omega_{0}\left(h, h^{-1} g\right)\right|\left|f_{t}(h)\right|\left|f_{t}^{\prime}\left(h^{-1} g\right)\right|\right)^{2}(1+\ell(g))^{2 s} .
$$

Let $C$ and $M$ be the constants controlling the growth of $\omega_{0}$. If we put $\tilde{f}_{t}(g)=f_{t}(g)(1+\ell(g))^{M}$, the above is bounded by

$$
C\left\||\tilde{f}| *\left|\tilde{f}^{\prime}\right|\right\|_{\ell, s}^{2} \leq C K(s)^{2}\||\tilde{f}|\|_{\ell, s}\left\|\left|\tilde{f}^{\prime}\right|\right\|_{\ell, s}=C K(s)^{2}\|f\|_{\ell, s+\frac{M}{2}}^{2}\left\|f^{\prime}\right\|_{\ell, s+\frac{M}{2}}^{2} .
$$

This shows that $f *_{\omega^{*}} f^{\prime}$ indeed has a bounded first order derivative.

Let us now proceed to the higher order derivatives. For $f \in C^{\infty}\left(I ; H_{\ell}^{\infty}(G)\right)$, again put

$$
\|f\|_{m}=\sum_{k=0}^{m} \frac{1}{k !} \max _{s \in I}\left\|\partial_{t}^{k} f_{s}\right\|_{\ell, m} .
$$

When $f^{1}, \ldots, f^{n}$ in $C^{\infty}\left(I ; H_{\ell}^{\infty}(G)\right)$, instead of (5.5) we claim

$$
\left\|f^{1} \cdots f^{n}\right\|_{m} \leq\left(K\left(m_{+}\right) e^{C}\right)^{n}\left\|f^{1}\right\|_{m_{+}} \cdots\left\|f^{n}\right\|_{m_{+}}
$$

with $m_{+}=m\left(1+\frac{M}{2}\right)$ this time. For $\left(g_{1}, \ldots, g_{n}\right) \in G^{n}$, put

$$
\omega_{0}^{(n)}\left(g_{1}, \ldots, g_{n}\right)=\omega_{0}\left(g_{1}, g_{2}\right)+\omega_{0}\left(g_{1} g_{2}, g_{3}\right)+\cdots+\omega_{0}\left(g_{1} \cdots g_{n-1}, g_{n}\right) .
$$

Then, with the product $h=f^{1} \cdots f^{n}$ in $\Gamma\left(I ; C_{r, \omega^{*}}^{*}(G)\right)$, we can bound $\frac{1}{k !} \max _{s}\left\|\partial_{t}^{k} h_{s}\right\|_{\ell, m}$ by

$$
\begin{gathered}
\sum_{\substack{a_{1}+\cdots+a_{n}+b_{1} \\
+\cdots+b_{n-1}=k}} \frac{K\left(m+\left(b_{1}+\cdots+b_{n-1}\right) \frac{M}{2}\right)^{n} \mathrm{C}^{b_{1}+\cdots+b_{n-1}}}{a_{1} ! \cdots a_{n} ! b_{1} ! \cdots b_{n-1} !}\left\|\partial_{t}^{a_{1}} f_{s}^{1}\right\|_{\ell, m+\left(b_{1}+\cdots+b_{n-1}\right) \frac{M}{2}} \\
\times\left\|\partial_{t}^{a_{2}} f_{s}^{2}\right\|_{\ell, m+\left(b_{1}+\cdots+b_{n-1}\right) \frac{M}{2}} \cdots\left\|\partial_{t}^{a_{n}} f_{s}^{n}\right\|_{\ell, m+b_{n-1} \frac{M}{2}}
\end{gathered}
$$

repeatedly using the estimates of the form (5.8). Then, using $b_{1}+\cdots+b_{n-1} \leq k \leq m$ and $\sum_{i} C^{b_{i}} / b_{i} ! \leq e^{C}$, we obtain the desired estimate $(5.9)$. 
The rest proceeds as in the proof of [33. Lemma 1.2]. Namely, having the $m$-convexity is equivalent to finding a system of convex neighborhoods of 0 which are idempotent in the sense that $U \cdot U \subset U$. Putting $B_{m}(r)=\left\{f \mid\|f\|_{m}<r\right\} \subset C^{\infty}\left(I ; H_{\ell}^{\infty}(G)\right)$, our claim above implies

$$
B_{m\left(1+\frac{M}{2}\right)}\left(K\left(m_{+}\right)^{-1} e^{-C}\right)^{n} \subset B_{m}(1)
$$

for any $n$. Thus, the convex hull $U_{m}$ of the $B_{m_{+}}\left(K\left(m_{+}\right)^{-1} e^{-C}\right)^{n}$ for $n=1,2, \ldots$ is a neighborhood of 0 satisfying $U_{m}^{2}=U_{m}$. Then $\frac{1}{m^{\prime}} U_{m}$ for $m, m^{\prime}=1,2, \ldots$ is a fundamental system of idempotent neighborhoods.

Proposition 5.7. Under the same assumption as Proposition 5.6. $C^{\infty}\left(I ; H_{\ell}^{\infty}(G)\right)$ is a spectral subalgebra of $\Gamma\left(I ; C_{r, \omega^{*}}^{*}(G)\right)$.

Proof. First we claim that $C\left(I, H_{\ell}^{\infty}(G)\right)$, or equivalently $C\left(I, H_{\ell}^{m}(G)\right)$ when $m$ is sufficiently large, is a spectral subalgebra of $\Gamma\left(I ; C_{r, \omega^{*}}^{*}(G)\right)$. The proof for each fiber [31, Proposition 6.11] carries on to this case, by replacing the norm of $\ell_{2}(G)$ by that of the Hilbert $\Gamma\left(I ; C_{r, \omega^{*}}^{*}(G)\right)$-module $C\left(I ; \ell_{2}(G)\right)$. (Otherwise we can appeal to 35. .)

The rest of proof is analogous to the second half of Proposition 5.2 . Suppose that $f \in C^{\infty}\left(I ; H_{\ell}^{\infty}(G)\right)$ is invertible in $C\left(I, H_{\ell}^{\infty}(G)\right)$. The assertion follows if we can show that $f^{-1}$ belongs to $C^{m}\left(I ; H_{\ell}^{m}(G)\right)$ when $m$ is sufficiently large. Let us take an element $f^{\prime} \in C^{m}\left(I, H_{\ell}^{m}(G)\right)$ satisfying

$$
\left\|f^{-1}-f^{\prime}\right\|_{C\left(I, H_{\ell}^{m_{+}}(G)\right)}<C_{1}\left(K\left(m_{+}\right) e^{C}\|f\|_{C\left(I, H_{\ell}^{m_{+}}(G)\right)}\right)^{-1}
$$

for some $0<C_{1}<1$. Thus, we have $\|x\|_{C\left(I, H_{\ell}^{m_{+}}(G)\right)}<C_{1} K\left(m_{+}\right)^{-1} e^{-C}$ for $x=1-f^{\prime} f$. Using this we are going to show that $\left\|x^{n}\right\|_{m}=o\left(C_{2}^{n}\right)$ for any $C_{1}<C_{2}<1$, which implies that $(1-x)^{-1}$, hence $f^{-1}=(1-x)^{-1} f^{\prime}$ also, is in $C^{m}\left(I, H_{\ell}^{m}(G)\right)$.

Replacing $f^{1}, \ldots, f^{n}$ in the proof of Proposition 5.6 by $x$, from 5.10 we can bound $\frac{1}{k !} \max _{s \in I}\left\|\partial_{t}^{k} x_{s}^{n}\right\|_{\ell, m}$ from above by

$$
\sum_{a_{1}+\cdots+a_{n} \leq k} \frac{K\left(m_{+}\right)^{n} e^{n C}}{a_{1} ! \cdots a_{n} !}\left\|\partial_{t}^{a_{1}} x_{s}\right\|_{\ell, m_{+}} \cdots\left\|\partial_{t}^{a_{n}} x_{s}\right\|_{\ell, m_{+}} .
$$

Now, let us suppose $n>m$ so that the $a_{i}$ start to contain 0 . f we fix the possibility of the possible values of $a_{1}, \ldots, a_{n}$ up to permutation, the multiplicity in the above sum is bounded by

$$
n(n-1) \cdots(n-k+1) \leq n^{m} .
$$

Hence we have

$$
\left\|x^{n}\right\|_{m} \leq K\left(m_{+}\right)^{n} e^{n C} n^{m}\|x\|_{m_{+}}^{m}\|x\|_{C\left(I, H_{\ell} m_{+}(G)\right)}^{n-m} .
$$

By assumption on $\|x\|_{C\left(I, H_{\ell}^{m_{+}}(G)\right)}$, we indeed have that this is asymptotically bounded by $C_{2}^{n}$ as $n \rightarrow$ $\infty$.

5.3. Effective conjugacy problem. We now want to use the formula of Proposition 2.5. Let $\phi$ be a cyclic cocycle on $\mathcal{A}=S_{1}(G)$ or $H_{\ell}^{\infty}(G)$ supported on the conjugacy class of $x$. Take $\psi: G \rightarrow \sqrt{-1} \mathbb{R}$ as in Section 3, and consider $\phi^{(t)}$. Then, as follows from the precise form of $\xi$ given in (3.1), it extends to a cyclic cocycle on $\mathcal{A}_{\omega^{t}}=S_{1}\left(G, \omega^{t}\right)$ or $H_{\ell}^{\infty}\left(G, \omega^{t}\right)$ for each $t$.

If the growth of $\xi\left(g_{0} \cdots g_{n}\right)$ is bounded by some polynomial in $\ell\left(g_{0}\right), \ldots, \ell\left(g_{n}\right)$, the family $\left(\phi^{(t)}\right)_{t}$ is smooth in $t$ and $\theta$ in Section 3 makes sense as a functional on $\mathcal{A} \hat{\otimes} \mathcal{A}$. Since the growth $\omega_{0}(g, h)$ is bounded by some polynomial in $\ell(g)$ and $\ell(h)$, it is enough to know that $\ell\left(g^{g_{0}}\right)$ is bounded by some polynomial in $\ell\left(g_{0}\right)$ when $g_{0}$ is conjugate to $x$. Note also that if $C_{G}(x)$ is finite, the growth rate does not depend on the choice of $g^{g_{0}}$.

Such problem (usually without finiteness assumption on the order of $x$ ) is known as the effective conjugacy problem: consider the conjugacy length function

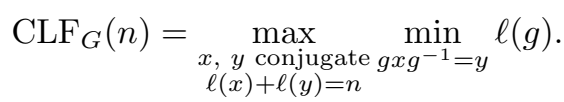

This is known to be at most linear for the hyperbolic groups [30], for the solvable groups of the form $\mathbb{Z}^{k} \rtimes_{A} \mathbb{Z}$ with $A \in \mathrm{SL}_{k}(\mathbb{Z})$ contained in a split torus $[44$, and for the torsion free 2-step nilpotent groups [19], as well as the crystallographic groups (see the next section). A quadratic bound is known for fundamental groups of prime 3-manifolds [2], see also [44, Theorem 5.5]. 
Recall that, in the first half of the next theorem, $G$ being 'good' means that it satisfies the BaumConnes conjecture with coefficients and that the natural map $K_{*}\left(\ell_{1}(G ; A)\right) \rightarrow K_{*}\left(G \ltimes_{r} A\right)$ is isomorphism for any $G$-C*-algebra $A$, which are satisfied by the Haagerup groups and the hyperbolic groups.

Theorem 5.8. Let $G$ be a good group with polynomially bounded conjugacy length function, and $\omega_{0}$ be an $\sqrt{-1} \mathbb{R}$-valued 2-cocycle of polynomial growth. Furthermore, let $x$ be a torsion element of $G$ such that $e^{t\left[\omega_{0}\right]}$ makes sense in $\mathrm{H}^{*}\left(C_{G}(x) ; \mathbb{C}\right)$. Then for any cyclic cocycle $\phi$ on $S_{1}(G)$ supported on the conjugacy class of $x$ and periodic cyclic cycle $c \in \mathrm{HP}_{*}\left(S_{1}\left(G ; C^{\infty}(I)\right)\right)$, the pairing

$$
\left\langle\mathrm{ev}_{t} c, \phi^{(t)} \cup e^{-t\left[\omega_{0}\right]}\right\rangle
$$

is constant in $t$, when $\phi^{(t)}$ is constructed from $\xi: G \rightarrow \sqrt{-1} \mathbb{R}$ as in Section 3.2.

An analogous statement holds for cyclic cocycles on $H_{\ell}^{\infty}(G)$ when $G$ is of rapid decay.

We thus have $\left\langle\operatorname{ev}_{t} c, \phi^{(t)}\right\rangle=\left\langle\operatorname{ev}_{0} c, \phi \cup e^{t\left[\omega_{0}\right]}\right\rangle$ in the above setting. Note also that, as we have seen in the previous section, the $K$-groups of $S_{1}\left(G ; C^{\infty}(I)\right)$ and $C_{r, \omega^{t}}^{*}(G)$ are naturally isomorphic, so this gives a concrete description of the pairing between $\operatorname{HC}^{*}\left(S_{1}\left(G, \omega^{t}\right)\right)$ and $K_{*}\left(C_{r, \omega^{t}}^{*}(G)\right)$ in terms of the one between $\mathrm{HC}^{*}\left(S_{1}(G)\right)$ and $K_{*}\left(C_{r}^{*}(G)\right)$.

\section{K-THEORY OF TWISTED CRYSTALLOGRAPHIC GROUP ALGEBRAS}

Let us consider a discrete $G$ sitting in an exact sequence $1 \rightarrow \mathbb{Z}^{n} \rightarrow G \rightarrow F \rightarrow 1$ such that $F$ is finite, and its induced action on $\mathbb{Z}^{n}$ is free away from origin. These groups are of polynomial growth, hence are amenable and there is no distinction between full and reduced twisted groups algebras. We also know that various classes of rapid decay functions coincide [21], so we just write $S(G)$. By [43, Corollary 2.3.18] the conjugacy length function of $G$ has a linear growth. Moreover, one may take $\mathbb{R}^{n}$ as a model of $\underline{E} G\left[9\right.$, hence $G \backslash \mathbb{R}^{n}$ as a one for $\underline{B} G=G \backslash \underline{E} G$. Any $\sqrt{-1} \mathbb{R}$-valued 2-cocycle is up to cohomology represented by an $F$-invariant 2-cocycle on $\mathbb{Z}^{n}$.

Example 6.1. Let $G$ be the group $\mathbb{Z}^{n}$. For each $n \times n$ real skew-symmetric matrix $\theta$, we can construct a 2-cocycle on this group by defining $\omega_{\theta}(x, y)=\exp (\sqrt{-1}\langle\theta x, y\rangle)$. The corresponding twisted group $\mathrm{C}^{*}$-algebra $\mathrm{C}^{*}\left(G, \omega_{\theta}\right)$ is also called the noncommutative torus $C\left(\mathbb{T}_{\theta}^{n}\right)$. For $n=2$, since $\theta$ depends on only one number $\theta^{\prime}$ as $\langle\theta x, y\rangle=\frac{\theta^{\prime}}{2}\left(x_{2} y_{1}-x_{1} y_{2}\right)$, we call that number $\theta$ also.

Example 6.2. Let $F$ be a finite subgroup of $\mathrm{GL}_{n}(\mathbb{Z})$ such that each $W \in F$ leaves $\theta$ invariant, i.e., $W^{T} \theta W=\theta$. Then we can define a 2-cocycle $\omega_{\theta}^{\prime}$ on $G=\mathbb{Z}^{n} \rtimes F$ by $\omega_{\theta}^{\prime}((x, g),(y, h))=\omega_{\theta}(x, g \cdot y)$ for $x, y \in \mathbb{Z}^{n}$ and $g, h \in F$. If each $W \in F$ not identity acts free away from origin, we obtain a crystallographic group. Again note that when $n=2$, any finite subgroup of $\mathrm{SL}_{2}(\mathbb{Z})$ will do.

Let $\mathcal{M}$ denote the set of conjugacy classes of maximal finite subgroups of $G$. In the following theorem, $\tilde{K}_{0}(\mathbb{C}[P])$ denotes the reduced $K_{0}$-group, that is, the kernel of the map $K_{0}(\mathbb{C}[P]) \rightarrow K_{0}(\mathbb{C})$ induced by the trivial representation (the augmentation map $\mathbb{C}[P] \rightarrow \mathbb{C}, \lambda_{g} \mapsto 1$ ). Note also that the map $K_{i}\left(C^{*}\left(\mathbb{Z}^{n}\right)\right) \rightarrow K_{i}\left(C^{*}(G)\right)$ induced by the inclusion $\mathbb{Z}^{n} \rightarrow G$ factors through the space of coinvariants $\mathbb{Z} \otimes_{F} K_{i}\left(C^{*}\left(\mathbb{Z}^{n}\right)\right)$.

Theorem $6.3([26])$. In the even degree, there exists an exact sequence

$$
0 \longrightarrow \bigoplus_{P \in \mathcal{M}} \tilde{K}_{0}(\mathbb{C}[P]) \longrightarrow K_{0}\left(C^{*}(G)\right) \longrightarrow K_{0}(\underline{B} G) \longrightarrow 0
$$

which splits after inverting $|F|$. The natural map

$$
\mathbb{Z} \otimes_{F} K_{0}\left(C^{*}\left(\mathbb{Z}^{n}\right)\right) \oplus\left(\bigoplus_{P \in \mathcal{M}} \tilde{K}_{0}(\mathbb{C}[P])\right) \rightarrow K_{0}\left(C^{*}(G)\right)
$$

also becomes a bijection after inverting $|F|$. In the odd degree, we have $K_{1}\left(C^{*}(G)\right) \simeq K_{1}(\underline{B} G)$.

Under the above setting, any nontrivial finite subgroup of $G$ has a finite normalizer 29, Lemma 6.1]. In particular, any nontrivial torsion element has a finite centralizer. Thus, for the twisted group algebras, the pairing with cyclic cocycles supported by the conjugacy class of such an elements is described by Section 3.1 .

For the above examples $\mathrm{H}^{*}(G ; \mathbb{C})$ is bounded in degree by $n$. Hence the assumptions of Theorem 5.8 are satisfied and we can compute the pairings $\left\langle\operatorname{ev}_{t} c, \phi^{(t)}\right\rangle$ for $c \in \operatorname{HP}_{*}\left(S_{1}\left(G ; C^{\infty}(I)\right)\right)$. 
For $x=e$, the pairing reduces to that between

$$
K^{*}\left(B \mathbb{Z}^{n}\right) \simeq K^{*}\left(\mathbb{T}^{n}\right), \quad \quad \operatorname{HP}^{*}\left(C^{\infty}\left(\mathbb{T}^{n}\right)\right) \simeq \bigoplus_{k} \mathrm{H}_{*+2 k}\left(\mathbb{T}^{n} ; \mathbb{C}\right)
$$

with modification by $e^{t\left[\omega_{0}\right]}$ with $\left[\omega_{0}\right] \in \mathrm{H}^{2}\left(\mathbb{T}^{n} ; \sqrt{-1} \mathbb{R}\right)$. For the even degree, on the image of $K_{0}\left(C^{*}\left(\mathbb{Z}^{n}\right)\right)$ it reduces to the pairing between

$$
\left(\bigwedge^{2 *} \mathbb{C}^{n}\right)^{F} \simeq \mathrm{H}^{2 *}\left(\mathbb{Z}^{n} ; \mathbb{C}\right)^{F} \simeq \mathrm{H}^{2 *}\left(B \mathbb{Z}^{n} ; \mathbb{C}\right)^{F}
$$

and $\mathbb{Z} \otimes_{F} K_{0}\left(C^{*}\left(\mathbb{Z}^{n}\right)\right) \simeq \mathbb{Z} \otimes_{F} \mathrm{H}_{2 *}\left(B \mathbb{Z}^{n} ; \mathbb{Z}\right)$. On the image of $\tilde{K}_{0}(\mathbb{C}[P])$, we just pick up the coefficient of $e \in G$ in the projections representing the $\tilde{K}_{0}$-classes. For the odd degree it reduces to the natural pairing of $\left(\bigwedge^{2 *+1} \mathbb{C}^{n}\right)^{F} \simeq \mathrm{H}^{2 *+1}\left(\mathbb{Z}^{n} ; \mathbb{C}\right)^{F}$ and $K_{1}(\underline{B} G) \simeq K_{1}(B G)$.

On the other hand, for nontrivial torsion element $x$, the pairing with $\tau_{\omega^{t}}^{x}$ essentially does not see $t$.

If $F=\mathbb{Z}_{m}$, the kernel of natural map $\mathbb{Z} \otimes_{F} K_{1}\left(C^{*}\left(\mathbb{Z}^{n}\right)\right) \rightarrow K_{1}\left(C^{*}(G)\right)$ is annihilated by the multiplication by $m$, the $K$-groups of $\mathrm{C}^{*}(G)$ are free commutative groups whose rank can be explicitly described [26]. Let us further assume that $p=m$ is prime. Then any non-trivial finite subgroup $P$ of $G$ must be isomorphic to $\mathbb{Z}_{p}$ via the restriction of the projection map $G \rightarrow \mathbb{Z}_{p}$. Thus any nontrivial finite subgroup of $G$ represents an element of $\mathcal{M}$. We have the following precise description of the $K$-groups.

Theorem $6.4([1])$. The number $n^{\prime}=n /(p-1)$ is an integer, and $|\mathcal{M}|=p^{n^{\prime}}$. The rank of K-homology groups of $\underline{B} G$ are given by

$$
\operatorname{rk} K_{0}(\underline{B} G)=\frac{2^{n}+p-1}{2 p}+\frac{(p-1) p^{n^{\prime}-1}}{2}, \quad \operatorname{rk} K_{1}(\underline{B} G)=\frac{2^{n}+p-1}{2 p}-\frac{(p-1) p^{n^{\prime}-1}}{2} .
$$

We want to explain how projections in $\tilde{K}_{0}(\mathbb{C}[P])$ contribute to the $K_{0}\left(C^{*}(G)\right.$ and $K_{0}\left(C^{*}\left(G, \omega_{\theta}^{\prime}\right)\right)$. Since $\mathbb{C}[P]$ is isomorphic to the algebra $C(\hat{P}) \simeq \mathbb{C}^{p}, K_{0}(\mathbb{C}[P])$ is the free abelian group of rank $p$. Now let $g$ be a generator of $P$. The minimal projections of $\mathbb{C}[P]$ are given by

$$
Q_{j, g}=\frac{1}{p} \sum_{k=0}^{p-1} \exp \left(\sqrt{-1} \frac{2 \pi}{p} j k\right) \lambda_{g^{k}}
$$

for $j=0, \cdots, p-1$, which also represent a basis of $K_{0}(\mathbb{C}[P])$. Since $Q_{0, g}$ represents the trivial representation, a basis of $\tilde{K}_{0}(\mathbb{C}[P])$ is given by $Q_{1, g}, \cdots, Q_{p-1, g}$.

By the above theorem, these projections are still linearly independent in $K_{0}\left(C^{*}(G)\right)$. To get elements of $K_{0}\left(C^{*}\left(G, \omega_{\theta}^{\prime}\right)\right)$, we need to modify this presentation a bit. Continuing to denote a generator of $P$ by $g$ the unitary $\lambda_{g}$ has order $p$ in $C^{*}(G)$. But since we modified the product in $C^{*}\left(G, \omega_{\theta}^{\prime}\right)$, the unitary $\lambda_{g}^{\left(\omega_{\theta}^{\prime}\right)}$ need not be so. Still, since $\omega_{\theta}^{\prime}$ is cohomologically trivial on the finite group $P$ and hence $\mathbb{C}_{\omega_{\theta}^{\prime}}[P] \simeq \mathbb{C}[P]$, we can always multiply a suitable $z \in \mathbb{T}$ so that order of the unitary $z \lambda_{g}^{\left(\omega_{\theta}^{\prime}\right)}$ is $p$. Then a similar formula

$$
Q_{j, g}^{(\theta)}=\frac{1}{p} \sum_{k=0}^{p-1} \exp \left(\sqrt{-1} \frac{2 \pi}{p} j k\right) z^{k} \lambda_{g^{k}}^{\left(\omega_{\theta}^{\prime}\right)}
$$

for $j=1, \cdots, p-1$ will give projections which are elements of $K_{0}\left(C^{*}\left(G, \omega_{\theta}^{\prime}\right)\right)$.

Remark 6.5. Yashinski [55] proposed another approach to compare $\operatorname{HP}_{*}\left(S\left(\mathbb{Z}^{n}, \omega_{\theta}\right)\right)$ for different $\theta$ based on $A_{\infty}$-isomorphism of Cartan-Eilenberg complexes, which is equivariant under the action of $\mathbb{Z}_{m}$ as above and would provide alternative route to the above result. While this is an interesting viewpoint, our understanding is that there is a gap in his argument, mainly because the cyclic bicomplex is not functorial for $A_{\infty}$-morphisms.

6.1. Crossed product of $\mathbb{Z}^{2}$ by $\mathbb{Z}_{3}$. Let us come back to Example 2.4. and compare it to [5]. This group $\mathbb{Z}^{2} \rtimes \mathbb{Z}_{3}$ can be presented as

$$
G=\left\langle u, v, w \mid w^{3}=e, u v=v u, w u w^{-1}=u^{-1} v, w v w^{-1}=u^{-1}\right\rangle .
$$

Up to conjugation, the finite subgroups of $G$ are generated by one of the elements $w, u w$ or, $u^{2} w$ all of which are of order 3 . Since $\omega_{\theta}^{\prime}(w, w)=1$, the first element still has order 3 in the twisted group algebra, and the corresponding projections $Q_{1, w}^{(\theta)}$ and $Q_{2, w}^{(\theta)}$ give nontrivial classes $K_{0}\left(C^{*}\left(G, \omega_{\theta}^{\prime}\right)\right)$. On the other hand,

$$
\omega_{\theta}^{\prime}(u w, u w) \omega_{\theta}^{\prime}(u w u w, u w)=\omega_{\theta}\left(u, u^{-1} v\right) \omega_{\theta}\left(v, v^{-1}\right)=e^{-\sqrt{-1} \frac{\theta}{2}}
$$


shows that $\lambda_{u w}^{\left(\omega_{\theta}^{\prime}\right)}$ is not of order 3. But an adjustment like $\exp \left(\sqrt{-1} \frac{\theta}{6}\right) \lambda_{u w}^{\left(\omega_{\theta}^{\prime}\right)}$ gives the right order. When $x, g \in G$ are torsion elements and the intersection $\operatorname{Ad}_{G}(x) \cap\langle g\rangle$ is nontrivial, there is a nontrivial pairing between $Q_{j, g}^{(\theta)}$ and $\tau_{\omega_{\theta}^{\prime}}^{x}$. Theorem 3.4 says that, due to an intricate way the coefficients in both are modified by $\theta$, the value of $\tau_{\omega_{\theta}^{\prime}}^{x}\left(Q_{j, g}^{(\theta)}\right)$ remains constant.

For simplicity, let us write $e(t)$ instead of $\exp (\sqrt{-1} t \theta)$. Then as noticed above $e\left(\frac{1}{6}\right) \lambda_{u w}^{\left(\omega_{\theta}^{\prime}\right)}$ is an unitary of order 3. Similarly one can see that $e\left(\frac{2}{3}\right) \lambda_{u^{2} w}^{\left(\omega_{\theta}^{\prime}\right)}$ is also an unitary of order 3 . Then we have the projections $Q_{1, w}^{(\theta)}, Q_{2, w}^{(\theta)}, Q_{1, u w}^{(\theta)}, Q_{2, u w}^{(\theta)}, Q_{1, u^{2} w}^{(\theta)}, Q_{2, u^{2} t}^{(\theta)}$ defining nontrivial classes in $K_{0}\left(C^{*}\left(G, \omega_{\theta}^{\prime}\right)\right)$.

Recall from [5] that if $\alpha$ defines an action of $\mathbb{Z}_{p}$ on $A$, an $\alpha$-invariant functional $\phi$ on $A$ is said to be an $\alpha$-trace if

$$
\phi(x y)=\phi(\alpha(y) x)
$$

holds for any $x, y \in A$. An $\alpha^{s}$-trace $\phi$ gives rise to a trace $T_{\phi}$ on $\mathbb{Z}_{p} \ltimes A$ defined by

$$
T_{\phi}\left(x_{0}+x_{1} w+\cdots+x_{p-1} w^{p-1}\right)=\phi\left(x_{p-s}\right)
$$

for $x_{i} \in A$ and $w$ being the copy of $1 \in \mathbb{Z}_{p}$.

Coming back to the example, let us consider the induced action of $\mathbb{Z}_{3}$ on $S\left(\mathbb{Z}^{2}, \omega_{\theta}\right)$. From 5 , Theorem 3.3 , we have following $\alpha$-traces $\phi_{l}^{1}$ on $S\left(\mathbb{Z}^{2}, \omega_{\theta}\right)$ :

$$
\phi_{l}^{1}\left(\lambda_{u}^{\left(\omega_{\theta}\right) m} \lambda_{v}^{\left(\omega_{\theta}\right) n}\right)=e\left(\frac{1}{6}\left((m-n)^{2}-l^{2}\right)\right) \delta_{3 \mathbb{Z}}(m-n-l)
$$

for $l=0,1,2$. Similarly we have following $\alpha^{2}$-traces $\phi_{l}^{2}$ on $S\left(\mathbb{Z}^{2}, \omega_{\theta}\right)$ :

$$
\phi_{l}^{2}\left(\lambda_{u}^{\left(\omega_{\theta}\right) m} \lambda_{v}^{\left(\omega_{\theta}\right) n}\right)=e\left(-m n-\frac{1}{6}\left((m-n)^{2}-l^{2}\right)\right) \delta_{3 \mathbb{Z}}(m-n-l)
$$

for $l=0,1,2$. Each $\phi_{l}^{i}$ then gives the the traces $T_{l}^{i}=T_{\phi_{l}^{i}}$ on $\mathbb{Z}_{3} \ltimes S\left(\mathbb{Z}^{2}, \omega_{\theta}\right)=S\left(G, \omega_{\theta}^{\prime}\right)$. Using $\lambda_{u}^{\left(\omega_{\theta}\right) m} \lambda_{v}^{\left(\omega_{\theta}\right) n}=e\left(-\frac{m n}{2}\right) \lambda_{u^{m} v^{n}}^{\left(\omega_{\theta}\right)}$, it is straightforward to check that the trace $\tau_{\omega_{\theta}^{\prime}}^{w}$ of Example 2.4 is exactly equal to $T_{0}^{2}$. (We do not see the effect of $\psi$ discussed in Section 3 because $\omega_{0}(y, w)=0$ for any $y \in G$.)

The pairing of the above projections and traces are given by the following table (cf. [5. Theorem 1.2]), which is independent of $\theta$ as suggested by Theorem 3.4

\begin{tabular}{l|r|r|r|r|r|r} 
& $\tau_{\omega_{\theta}^{\prime}}^{w^{2}}=T_{0}^{1}$ & $\tau_{\omega_{\theta}^{\prime}}^{u w^{2}}=T_{1}^{1}$ & $\tau_{\omega_{\theta}^{\prime}}^{u^{2} w^{2}}=T_{2}^{1}$ & $\tau_{\omega_{\theta}^{\prime}}^{w}=T_{0}^{2}$ & $\tau_{\omega_{\theta}^{\prime}}^{u w}=T_{1}^{2}$ & $\tau_{\omega_{\theta}^{\prime}}^{u^{2} w}=T_{2}^{2}$ \\
\hline$Q_{1, w}^{(\theta)}$ & $\frac{1}{3} e^{\frac{4 \pi}{3} \sqrt{-1}}$ & 0 & 0 & $\frac{1}{3} e^{\frac{2 \pi}{3} \sqrt{-1}}$ & 0 & 0 \\
$Q_{2, w}^{(\theta)}$ & $\frac{1}{3} e^{\frac{2 \pi}{3} \sqrt{-1}}$ & 0 & 0 & $\frac{1}{3} e^{\frac{4 \pi}{3} \sqrt{-1}}$ & 0 & 0 \\
$Q_{1, u w}^{(\theta)}$ & 0 & 0 & $\frac{1}{3} e^{\frac{4 \pi}{3} \sqrt{-1}}$ & 0 & $\frac{1}{3} e^{\frac{2 \pi}{3} \sqrt{-1}}$ \\
$Q_{2, u w}^{(\theta)}$ & 0 & 0 & $\frac{1}{3} e^{\frac{2 \pi}{3} \sqrt{-1}}$ & 0 & $\frac{1}{3} e^{\frac{4 \pi}{3} \sqrt{-1}}$ & 0 \\
$Q_{1, u^{2} w}^{(\theta)}$ & 0 & $\frac{1}{3} e^{\frac{4 \pi}{3} \sqrt{-1}}$ & 0 & 0 & 0 & $\frac{1}{3} e^{\frac{2 \pi}{3} \sqrt{-1}}$ \\
$Q_{2, u^{2} w}^{(\theta)}$ & 0 & $\frac{1}{3} e^{\frac{2 \pi}{3} \sqrt{-1}}$ & 0 & 0 & 0 & $\frac{1}{3} e^{\frac{4 \pi}{3} \sqrt{-1}}$
\end{tabular}

\section{REFERENCES}

[1] M. André, Le $d_{2}$ de la suite spectrale en cohomologie des groupes, C. R. Acad. Sci. Paris 260 (1965), 2669-2671 (French). MR0197526

[2] J. Behrstock and C. Druţu, Divergence, thick groups, and short conjugators, Illinois J. Math. 58 (2014), no. 4, 939-980. MR3421592

[3] R. A. Bonic, Symmetry in group algebras of discrete groups, Pacific J. Math. 11 (1961), 73-94. MR0120529

[4] K. S. Brown. (1994). Cohomology of groups, Graduate Texts in Mathematics, vol. 87, Springer-Verlag, New York, ISBN 0-387-90688-6. Corrected reprint of the 1982 original. MR1324339 (96a:20072)

[5] J. Buck and S. Walters, Connes-Chern characters of hexic and cubic modules, J. Operator Theory 57 (2007), no. 1, 35-65. MR2301936

[6] D. Burghelea, The cyclic homology of the group rings, Comment. Math. Helv. 60 (1985), no. 3, 354-365, DOI: $10.1007 / \mathrm{BF} 02567420$ MR814144 (88e:18007)

[7] X. Chen and S. Wei, Spectral invariant subalgebras of reduced crossed product $\mathrm{C}^{*}$-algebras, J. Funct. Anal. 197 (2003), no. 1, 228-246, DOI:10.1016/S0022-1236(02)00031-9. MR1957682

[8] A. Connes, Noncommutative differential geometry, Inst. Hautes Études Sci. Publ. Math. 62 (1985), 257-360. MR823176 (87i:58162)

[9] F. Connolly and T. Koźniewski, Rigidity and crystallographic groups. I, Invent. Math. 99 (1990), no. 1, 25-48, DOI:10.1007/BF01234410 MR1029389 
[10] J. Cuntz, Cyclic Theory, Bivariant K-Theory and the Bivariant Chern-Connes Character, Cyclic homology in noncommutative geometry, 2004, pp. 1-72, Springer-Verlag, Berlin. Operator Algebras and Non-commutative Geometry, II. MR2052770 (2005k:19008)

[11] J. F. Davis and W. Lück, The topological K-theory of certain crystallographic groups, J. Noncommut. Geom. 7 (2013), no. 2, 373-431, DOI:10.4171/JNCG/121. MR3054301

[12] S. Echterhoff, W. Lück, N. C. Phillips, and S. Walters, The structure of crossed products of irrational rotation algebras by finite subgroups of $\mathrm{SL}_{2}(\mathbb{Z})$, J. Reine Angew. Math. 639 (2010), 173-221, DOI:10.1515/CRELLE.2010.015, arXiv:math/0609784. MR/2608195 (2011c:46127)

[13] G. A. Elliott, On the $K$-theory of the $C^{*}$-algebra generated by a projective representation of a torsion-free discrete abelian group, Operator algebras and group representations, Vol. I (Neptun, 1980), 1984, pp. 157-184, Pitman, Boston, MA. MR731772 (85m:46067)

[14] E. Getzler, Cartan homotopy formulas and the Gauss-Manin connection in cyclic homology, Quantum deformations of algebras and their representations (Ramat-Gan, 1991/1992; Rehovot, 1991/1992), 1993, pp. 65-78, Bar-Ilan Univ., Ramat Gan. MR1261901 (95c:19002)

[15] P. G. Goerss and J. F. Jardine. (1999). Simplicial homotopy theory, Progress in Mathematics, vol. 174, Birkhäuser Verlag, Basel, DOI:10.1007/978-3-0348-8707-6, ISBN 3-7643-6064-X. MR1711612

[16] N. Higson and G. Kasparov, E-theory and KK-theory for groups which act properly and isometrically on Hilbert space, Invent. Math. 144 (2001), no. 1, 23-74, DOI:10.1007/s002220000118 MR.1821144(2002k:19005)

[17] J. W. Jenkins, Symmetry and nonsymmetry in the group algebras of discrete groups, Pacific J. Math. 32 (1970), 131-145. MR0256185

[18] R. Ji, A module structure on cyclic cohomology of group graded algebras, K-Theory 7 (1993), no. 4, 369-399, DOI: $10.1007 / \mathrm{BF} 00962054$ MR 1246282

[19] R. Ji, C. Ogle, and B. Ramsey, Relatively hyperbolic groups, rapid decay algebras and a generalization of the Bass conjecture, J. Noncommut. Geom. 4 (2010), no. 1, 83-124, DOI:10.4171/JNCG/50. With an appendix by Ogle. MR.2575390

[20] R. Ji and L. B. Schweitzer, Spectral invariance of smooth crossed products, and rapid decay locally compact groups, $K$-Theory 10 (1996), no. 3, 283-305, DOI:10.1007/BF00538186 MR1394381 (97d:46079)

[21] P. Jolissaint, Rapidly decreasing functions in reduced $\mathrm{C}^{*}$-algebras of groups, Trans. Amer. Math. Soc. 317 (1990), no. 1, 167-196, DOI:10.2307/2001458 MR943303 (90d:22006)

[22] M. Karoubi, Homologie cyclique des groupes et des algèbres, C. R. Acad. Sci. Paris Sér. I Math. 297 (1983), no. 7, 381-384. MR732839 (85g:18012)

[23] V. Lafforgue, K-théorie bivariante pour les algèbres de Banach et conjecture de Baum-Connes, Invent. Math. 149 (2002), no. 1, 1-95, DOI:10.1007/s002220200213. MR.1914617(2003d:19008)

[24] V. Lafforgue, A proof of property (RD) for cocompact lattices of $\mathrm{SL}(3, \mathbf{R})$ and $\mathrm{SL}(3, \mathbf{C})$, J. Lie Theory 10 (2000), no. 2, 255-267. MR.1774859

[25] V. Lafforgue, La conjecture de Baum-Connes à coefficients pour les groupes hyperboliques, J. Noncommut. Geom. 6 (2012), no. 1, 1-197, DOI: 10.4171/JNCG/89 arXiv:1201.4653 [math.0A] MR.2874956

[26] M. Langer and W. Lück, Topological K-theory of the group $\mathrm{C}^{*}$-algebra of a semi-direct product $\mathbb{Z}^{n} \rtimes \mathbb{Z} / m$ for a free conjugation action, J. Topol. Anal. 4 (2012), no. 2, 121-172, DOI:10.1142/S1793525312500082 MR2949238

[27] J.-L. Loday. (1998). Cyclic homology, Second, Grundlehren der Mathematischen Wissenschaften [Fundamental Principles of Mathematical Sciences], vol. 301, Springer-Verlag, Berlin, ISBN 3-540-63074-0. Appendix E by María O. Ronco, Chapter 13 by the author in collaboration with Teimuraz Pirashvili. MR 1600246 (98h:16014)

[28] J.-L. Loday and D. Quillen, Cyclic homology and the Lie algebra homology of matrices, Comment. Math. Helv. 59 (1984), no. 4, 569-591. MR780077 (86i:17003)

[29] W. Lück and R. Stamm, Computations of K- and L-theory of cocompact planar groups, K-Theory 21 (2000), no. 3, 249-292, DOI : 10.1023/A:1026539221644. MR:1803230

[30] I. G. Lysënok, Some algorithmic properties of hyperbolic groups, Izv. Akad. Nauk SSSR Ser. Mat. 53 (1989), no. 4, 814-832, 912. translation in Math. USSR-Izv. 35 (1990), no. 1, 145-163. MR.1018749

[31] V. Mathai, Heat kernels and the range of the trace on completions of twisted group algebras, The ubiquitous heat kernel, 2006, pp. 321-345, Amer. Math. Soc., Providence, RI. With an appendix by Indira Chatterji. MR 2218025 (2007c:58034)

[32] B. S. Mitjagin, Approximate dimension and bases in nuclear spaces, Uspehi Mat. Nauk 16 (1961), no. 4 (100), 63-132. MR0152865

[33] B. Mitiagin, S. Rolewicz, and W. Żelazko, Entire functions in B B-algebras, Studia Math. 21 (1961/1962), 291-306. MR 0144222

[34] J. A. Packer and I. Raeburn, Twisted crossed products of C*-algebras, Math. Proc. Cambridge Philos. Soc. 106 (1989), no. 2, 293-311. MR1002543 (90g:46097)

[35] W. Paravicini, The spectral radius in $\mathcal{C}_{0}(X)$-Banach algebras, J. Noncommut. Geom. 7 (2013), no. 1, 135-147, DOI : $10.4171 /$ JNCG/111 MR 3032813

[36] N. C. Phillips, K-theory for Fréchet algebras, Internat. J. Math. 2 (1991), no. 1, 77-129, DOI: 10.1142/S0129167X91000077 MR 1082838 (92e:46143)

[37] M. Pimsner and D. Voiculescu, Imbedding the irrational rotation $C^{*}$-algebra into an AF-algebra, J. Operator Theory 4 (1980), no. 2, 201-210. MR595412 (82d:46086)

[38] M. Puschnigg, New holomorphically closed subalgebras of $\mathrm{C}^{*}$-algebras of hyperbolic groups, Geom. Funct. Anal. 20 (2010), no. 1, 243-259, DOI:10.1007/s00039-010-0062-y. MR2647141

[39] S. Quddus, Cohomology of $\mathcal{A}_{\theta}^{\text {alg }} \rtimes \mathbb{Z}_{2}$ and its chern-connes pairing. preprint (2014), to appear in J. Noncommut. Geom.

[40] S. Quddus, Hochschild and cyclic homology of the crossed product of algebraic irrational rotational algebra by finite subgroups of $S L(2, \mathbb{Z})$, J. Algebra 447 (2016), 322-366, DOI:10.1016/j.jalgebra.2015.08.019. MR3427637 
[41] M. A. Rieffel, Irrational rotation $C^{*}$-algebras. short communication (1978), presented at International Congress of Mathematicians, Helsinki.

[42] G. S. Rinehart, Differential forms on general commutative algebras, Trans. Amer. Math. Soc. 108 (1963), $195-222$. MR0154906 (27 \#4850)

[43] A. W. Sale, The length of conjugators in solvable groups and lattices of semisimple lie groups, Ph.D. Thesis, University of Oxford, 2012.

[44] A. Sale, Conjugacy length in group extensions, Comm. Algebra 44 (2016), no. 2, 873-897, DOI : $10.1080 / 00927872.2014 .990021$ MR3449958

[45] L. B. Schweitzer, Dense m-convex Fréchet subalgebras of operator algebra crossed products by Lie groups, Internat. J. Math. 4 (1993), no. 4, 601-673, DOI:10.1142/S0129167X93000315. MR1232986 (94g:46058)

[46] L. B. Schweitzer, Spectral invariance of dense subalgebras of operator algebras, Internat. J. Math. 4 (1993), no. 2, 289-317, DOI:10.1142/S0129167X93000157 MR.1217384 (94i:46097)

[47] F. Trèves. (1967). Topological vector spaces, distributions and kernels, Academic Press, New York. MR0225131 (37 \#726)

[48] B. L. Tsygan, Homology of matrix Lie algebras over rings and the Hochschild homology, Uspekhi Mat. Nauk 38 (1983), no. 2(230), 217-218. Translation in Russ. Math. Survey 38(2) (1983), 198-199. MR695483 (85i:17014)

[49] B. Tsygan, Cyclic homology, Cyclic homology in non-commutative geometry, 2004, pp. 73-113, Springer-Verlag, Berlin. Operator Algebras and Non-commutative Geometry, II. MR2052770 (2005k:19008)

[50] A. Valette. (2002). Introduction to the Baum-Connes conjecture, Lectures in Mathematics ETH Zürich, Birkhäuser Verlag, Basel, ISBN 3-7643-6706-7. From notes taken by Indira Chatterji, With an appendix by Guido Mislin. MR/1907596 (2003f:58047)

[51] S. G. Walters, Projective modules over the non-commutative sphere, J. London Math. Soc. (2) 51 (1995), no. 3, 589-602, DOI: 10.1112/jlms/51.3.589 MR.1332894

[52] S. G. Walters, Chern characters of Fourier modules, Canad. J. Math. 52 (2000), no. 3, 633-672, DOI : 10.4153/CJM-2000-028-9 MR.1758235

[53] M. Yamashita, Deformation of algebras associated to group cocycles. preprint (2011), available at arXiv:1107.2512 [math.0A]

[54] M. Yamashita, Monodromy of Gauss-Manin connection for deformation by group cocycles. preprint (2012), available at arXiv:1207.6687 [math.KT] to appear in J. Noncommut. Geom.

[55] A. Yashinski, Periodic cyclic homology and smooth deformations, Ph.D. Thesis, The Pennsylvania State University, 2013. 